\title{
Positive solutions for a class of higher-order singular semipositone fractional differential systems with coupled integral boundary conditions and parameters
}

Ying Wang ${ }^{1,2}$, Lishan Liu' ${ }^{1 *}$ and Yonghong $\mathrm{Wu}^{3}$

${ }^{*}$ Correspondence:

Ils@mail.qfnu.edu.cn

'School of Mathematical Sciences,

Qufu Normal University, Qufu,

Shandong 273165, People's

Republic of China

Full list of author information is

available at the end of the article

\begin{abstract}
In this paper, we study the existence of a class of higher-order singular semipositone fractional differential systems with coupled integral boundary conditions and parameters. By using the properties of the Green's function and the Guo-Krasnosel'skii fixed point theorem, we obtain some existence results of positive solutions under some conditions concerning the nonlinear functions. The method of this paper is a unified method for establishing the existence of positive solutions for a large number of nonlinear differential equations with coupled boundary conditions. In the end, examples are given to demonstrate the validity of our main results.
\end{abstract}

MSC: 34B16; 34B18

Keywords: positive solutions; fractional differential system; coupled integral boundary conditions; singular

\section{Introduction}

Coupled boundary conditions arise in the study of reaction-diffusion equations, SturmLiouville problems, mathematical biology and so on; see [1-4]. Leung [5] studied the following reaction-diffusion system for prey-predator interaction:

$$
\begin{aligned}
& u_{t}(t, x)=\sigma_{1} \Delta u+u(a+f(u, v)), \quad t \geq 0, x \in \Omega \subset R^{n}, \\
& v_{t}(t, x)=\sigma_{2} \Delta v+v(-r+g(u, v)), \quad t \geq 0, x \in \Omega \subset R^{n},
\end{aligned}
$$

subject to the coupled boundary conditions

$$
\frac{\partial u}{\partial \eta}=0, \quad \frac{\partial v}{\partial \eta}-p(u)-q(v)=0 \quad \text { on } \partial \Omega,
$$

where $\triangle=\sum_{i=1}^{n} \frac{\partial^{2}}{\partial x_{i}^{2}}, a, r, \sigma_{1}, \sigma_{2}$ are positive constants, $f, g: R^{2} \rightarrow R$ have Hölder continuous partial derivatives up to second order in compact sets, $\eta$ is a unit outward normal at $\partial \Omega$ and $p$ and $q$ have Hölder continuous first derivatives in compact subsets of $[0,+\infty)$. The functions $u(t, x), v(t, x)$ respectively represent the density of prey and predator at time

\section{Springer}

(02014 Wang et al.; licensee Springer. This is an Open Access article distributed under the terms of the Creative Commons Attribution License (http://creativecommons.org/licenses/by/2.0), which permits unrestricted use, distribution, and reproduction in any medium, provided the original work is properly cited. 
$t \geq 0$ and at position $x=\left(x_{1}, \ldots, x_{n}\right)$. Similar coupled boundary conditions are also studied in [6] for a biochemical system.

Existence theory for boundary value problems of ordinary differential equations is well studied. However, differential equations with fractional order are a generalization of the ordinary differential equations to non-integer order. This generalization is not a mere mathematical curiosity but rather has interesting applications in many areas of science and engineering such as electrochemistry, control, porous media, electromagnetism, etc. There has been a significant development in the study of fractional differential equations in recent years; see, for example, [7-13]. Wang et al. [14] researched a coupled system of nonlinear fractional differential equations

$$
\left\{\begin{array}{l}
D_{0^{+}}^{\alpha} u(t)+f(t, v(t))=0, \\
D_{0^{+}}^{\beta} v(t)+g(t, u(t))=0, \quad 0<t<1, \\
u(0)=v(0)=0, \quad u(1)=a u(\xi), \quad v(1)=b v(\xi)
\end{array}\right.
$$

where $1<\alpha, \beta<2,0 \leq a, b<1,0<\xi<1, f, g:[0,1] \times[0,+\infty) \rightarrow[0,+\infty)$ are continuous functions, $D_{0^{+}}^{\alpha}, D_{0^{+}}^{\beta}$ are also two standard Riemann-Liouville fractional derivatives. By using the Banach fixed point theorem and nonlinear differentiation of Leray-Schauder type, the existence and uniqueness of positive solutions are obtained.

In [15], Yang considered the positive solutions to boundary values problem for a coupled system of nonlinear fractional differential equations as follows:

$$
\left\{\begin{array}{l}
D^{\alpha} u(t)+a(t) f(t, v(t))=0, \\
D^{\beta} v(t)+b(t) g(t, u(t))=0, \quad 0<t<1, \\
u(0)=0, \quad u(1)=\int_{0}^{1} \kappa(t) u(t) d t, \quad v(0)=0, \quad v(1)=\int_{0}^{1} \mu(t) v(t) d t,
\end{array}\right.
$$

where $1<\alpha, \beta \leq 2, a, b:(0,1) \rightarrow[0,+\infty)$ are continuous, $\kappa, \mu:[0,1] \rightarrow[0,+\infty)$ are nonnegative and integrable functions, $f, g:[0,1] \times[0,+\infty) \rightarrow[0,+\infty)$ are continuous, and $D^{\alpha}$, $D^{\beta}$ are standard Riemann-Liouville fractional derivatives. By applying the Banach fixed point theorem, nonlinear differentiation of Leray-Schauder type and the fixed point theorems of cone expansion and compression of norm type, sufficient conditions for the existence and nonexistence of positive solutions to a general class of integral boundary value problems for a coupled system of fractional differential equations are obtained.

Inspired by the above mentioned work and wide applications of coupled boundary conditions in various fields of sciences and engineering, in this paper, we research the existence result to a class of singular semipositone fractional differential systems with coupled integral boundary conditions of the type

$$
\left\{\begin{array}{l}
D_{0^{+}}^{\alpha_{1}} u(t)+\lambda_{1} f_{1}(t, u(t), v(t))=0, \\
D_{0^{+}}^{\alpha_{2}} v(t)+\lambda_{2} f_{2}(t, u(t), v(t))=0, \quad 0<t<1, \\
u(0)=u^{\prime}(0)=\cdots=u^{(n-2)}(0)=0, \quad u(1)=\mu_{1} \int_{0}^{1} v(s) d A_{1}(s), \\
v(0)=v^{\prime}(0)=\cdots=v^{(n-2)}(0)=0, \quad v(1)=\mu_{2} \int_{0}^{1} u(s) d A_{2}(s),
\end{array}\right.
$$

where $\lambda_{i}>0$ is a parameter, $n-1<\alpha_{i} \leq n, n \geq 2, D_{0^{+}}^{\alpha_{i}}$ is the standard Riemann-Liouville derivative. $\mu_{i}>0$ is a constant, $A_{i}$ is right continuous on $[0,1)$, left continuous at $t=1$, and nondecreasing on $[0,1], A_{i}(0)=0, \int_{0}^{1} x(s) d A_{i}(s)$ denotes the Riemann-Stieltjes integrals of $x$ with respect to $A_{i}, f_{i}:(0,1) \times[0,+\infty) \times[0,+\infty) \rightarrow(-\infty,+\infty)$ is a continuous function 
and may be singular at $t=0,1$ for $i=1,2$. By a positive solution of system (1.1), we mean that $(u, v) \in C[0,1] \times C[0,1],(u, v)$ satisfies $(1.1)$ and $u(t)>0, v(t)>0$ for all $t \in(0,1]$.

To the best knowledge of the authors, there is seldom earlier literature studying fractional differential system with coupled integral boundary conditions like system (1.1), especially when $f_{i}(t, u, v)(i=1,2)$ may be sign-changing, and may be singular at $t=0$ and $t=1$. Motivated by the results mentioned above, this paper attempts to fill part of this gap in the literature.

\section{Preliminaries and lemmas}

For convenience of the reader, we present some necessary definitions about fractional calculus theory.

Definition 2.1 $[16,17]$ Let $\alpha>0$ and let $u$ be piecewise continuous on $(0,+\infty)$ and integrable on any finite subinterval of $[0,+\infty)$. Then, for $t>0$, we call

$$
I_{0^{+}}^{\alpha} u(t)=\frac{1}{\Gamma(\alpha)} \int_{0}^{t}(t-s)^{\alpha-1} u(s) d s
$$

the Riemann-Liouville fractional integral of $u$ of order $\alpha$.

Definition 2.2 $[16,17]$ The Riemann-Liouville fractional derivative of order $\alpha>0, n-1 \leq$ $\alpha<n, n \in \mathbb{N}$ is defined as

$$
D_{0^{+}}^{\alpha} u(t)=\frac{1}{\Gamma(n-\alpha)}\left(\frac{d}{d t}\right)^{n} \int_{0}^{t}(t-s)^{n-\alpha-1} u(s) d s,
$$

where $\mathbb{N}$ denotes the natural number set, the function $u(t)$ is $n$ times continuously differentiable on $[0,+\infty)$.

Lemma $2.1[16,17]$ Let $\alpha>0$, if the fractional derivatives $D_{0^{+}}^{\alpha-1} u(t)$ and $D_{0^{+}}^{\alpha} u(t)$ are continuous on $[0,+\infty)$, then

$$
I_{0^{+}}^{\alpha} D_{0^{+}}^{\alpha} u(t)=u(t)+c_{1} t^{\alpha-1}+c_{2} t^{\alpha-2}+\cdots+c_{n} t^{\alpha-n},
$$

where $c_{1}, c_{2}, \ldots, c_{n} \in(-\infty,+\infty), n$ is the smallest integer greater than or equal to $\alpha$.

Lemma 2.2 Assume that the following condition $\left(\mathbf{H}_{0}\right)$ holds.

$\left(\mathbf{H}_{0}\right)$

$$
k_{1}=\int_{0}^{1} t^{\alpha_{2}-1} d A_{1}(t)>0, \quad k_{2}=\int_{0}^{1} t^{\alpha_{1}-1} d A_{2}(t)>0, \quad 1-\mu_{1} \mu_{2} k_{1} k_{2}>0 .
$$

Let $h_{i} \in C(0,1) \cap L(0,1)(i=1,2)$, then the system with the coupled boundary conditions

$$
\left\{\begin{array}{l}
D_{0^{+}}^{\alpha_{1}} u(t)+h_{1}(t)=0, \quad D_{0^{+}}^{\alpha_{2}} v(t)+h_{2}(t)=0, \quad 0<t<1, \\
u(0)=u^{\prime}(0)=\cdots=u^{(n-2)}(0)=0, \quad u(1)=\mu_{1} \int_{0}^{1} v(s) d A_{1}(s), \\
v(0)=v^{\prime}(0)=\cdots=v^{(n-2)}(0)=0, \quad v(1)=\mu_{2} \int_{0}^{1} u(s) d A_{2}(s)
\end{array}\right.
$$


has a unique integral representation

$$
\left\{\begin{array}{l}
u(t)=\int_{0}^{1} K_{1}(t, s) h_{1}(s) d s+\int_{0}^{1} H_{1}(t, s) h_{2}(s) d s, \\
v(t)=\int_{0}^{1} K_{2}(t, s) h_{2}(s) d s+\int_{0}^{1} H_{2}(t, s) h_{1}(s) d s
\end{array}\right.
$$

where

$$
\begin{aligned}
& K_{1}(t, s)=\frac{\mu_{1} \mu_{2} k_{1} t^{\alpha_{1}-1}}{1-\mu_{1} \mu_{2} k_{1} k_{2}} \int_{0}^{1} G_{1}(t, s) d A_{2}(t)+G_{1}(t, s), \\
& H_{1}(t, s)=\frac{\mu_{1} t^{\alpha_{1}-1}}{1-\mu_{1} \mu_{2} k_{1} k_{2}} \int_{0}^{1} G_{2}(t, s) d A_{1}(t), \\
& K_{2}(t, s)=\frac{\mu_{2} \mu_{1} k_{2} t^{\alpha_{2}-1}}{1-\mu_{1} \mu_{2} k_{1} k_{2}} \int_{0}^{1} G_{2}(t, s) d A_{1}(t)+G_{2}(t, s), \\
& H_{2}(t, s)=\frac{\mu_{2} t^{\alpha_{2}-1}}{1-\mu_{1} \mu_{2} k_{1} k_{2}} \int_{0}^{1} G_{1}(t, s) d A_{2}(t),
\end{aligned}
$$

and

$$
G_{i}(t, s)=\frac{1}{\Gamma\left(\alpha_{i}\right)}\left\{\begin{array}{ll}
{[t(1-s)]^{\alpha_{i}-1}-(t-s)^{\alpha_{i}-1},} & 0 \leq s \leq t \leq 1, \\
{[t(1-s)]^{\alpha_{i}-1},} & 0 \leq t \leq s \leq 1,
\end{array} \quad i=1,2 .\right.
$$

Proof System (2.1) is equivalent to the system of integral equations

$$
\begin{aligned}
& u(t)=u(1) t^{\alpha_{1}-1}+\int_{0}^{1} G_{1}(t, s) h_{1}(s) d s, \\
& v(t)=v(1) t^{\alpha_{2}-1}+\int_{0}^{1} G_{2}(t, s) h_{2}(s) d s .
\end{aligned}
$$

Integrating (2.4) and (2.5) with respect to $d A_{2}(t)$ and $d A_{1}(t)$ respectively, we have

$$
\begin{aligned}
& \int_{0}^{1} u(t) d A_{2}(t)=u(1) \int_{0}^{1} t^{\alpha_{1}-1} d A_{2}(t)+\int_{0}^{1} \int_{0}^{1} G_{1}(t, s) h_{1}(s) d s d A_{2}(t), \\
& \int_{0}^{1} v(t) d A_{1}(t)=v(1) \int_{0}^{1} t^{\alpha_{2}-1} d A_{1}(t)+\int_{0}^{1} \int_{0}^{1} G_{2}(t, s) h_{2}(s) d s d A_{1}(t) .
\end{aligned}
$$

Therefore, we can get

$$
\begin{aligned}
& \frac{1}{\mu_{1}} u(1)-k_{1} v(1)=\int_{0}^{1} \int_{0}^{1} G_{2}(t, s) h_{2}(s) d s d A_{1}(t), \\
& -k_{2} u(1)+\frac{1}{\mu_{2}} v(1)=\int_{0}^{1} \int_{0}^{1} G_{1}(t, s) h_{1}(s) d s d A_{2}(t) .
\end{aligned}
$$

Note that

$$
\left|\begin{array}{cc}
\frac{1}{\mu_{1}} & -k_{1} \\
-k_{2} & \frac{1}{\mu_{2}}
\end{array}\right|=\frac{1-\mu_{1} \mu_{2} k_{1} k_{2}}{\mu_{1} \mu_{2}} \neq 0
$$


Thus, system (2.6) and (2.7) has a unique solution for $u(1)$ and $v(1)$. By Cramer's rule and simple calculations, it follows that

$$
\begin{aligned}
u(1)= & \frac{\mu_{1}}{1-\mu_{1} \mu_{2} k_{1} k_{2}}\left(\int_{0}^{1} \int_{0}^{1} G_{2}(t, s) h_{2}(s) d s d A_{1}(t)\right. \\
& \left.+\mu_{2} k_{1} \int_{0}^{1} \int_{0}^{1} G_{1}(t, s) h_{1}(s) d s d A_{2}(t)\right) \\
v(1)= & \frac{\mu_{2}}{1-\mu_{1} \mu_{2} k_{1} k_{2}}\left(\int_{0}^{1} \int_{0}^{1} G_{1}(t, s) h_{1}(s) d s d A_{2}(t)\right. \\
& \left.+\mu_{1} k_{2} \int_{0}^{1} \int_{0}^{1} G_{2}(t, s) h_{2}(s) d s d A_{1}(t)\right) .
\end{aligned}
$$

Substituting (2.8) and (2.9) into (2.4) and (2.5), we have

$$
\begin{aligned}
u(t)= & \frac{\mu_{1} t^{\alpha_{1}-1}}{1-\mu_{1} \mu_{2} k_{1} k_{2}}\left(\int_{0}^{1} \int_{0}^{1} G_{2}(t, s) h_{2}(s) d s d A_{1}(t)\right. \\
& \left.+\mu_{2} k_{1} \int_{0}^{1} \int_{0}^{1} G_{1}(t, s) h_{1}(s) d s d A_{2}(t)\right)+\int_{0}^{1} G_{1}(t, s) h_{1}(s) d s \\
= & \int_{0}^{1} K_{1}(t, s) h_{1}(s) d s+\int_{0}^{1} H_{1}(t, s) h_{2}(s) d s, \\
v(t)= & \frac{\mu_{2} t^{\alpha_{2}-1}}{1-\mu_{1} \mu_{2} k_{1} k_{2}}\left(\int_{0}^{1} \int_{0}^{1} G_{1}(t, s) h_{1}(s) d s d A_{2}(t)\right. \\
& \left.+\mu_{1} k_{2} \int_{0}^{1} \int_{0}^{1} G_{2}(t, s) h_{2}(s) d s d A_{1}(t)\right)+\int_{0}^{1} G_{2}(t, s) h_{2}(s) d s \\
= & \int_{0}^{1} K_{2}(t, s) h_{2}(s) d s+\int_{0}^{1} H_{2}(t, s) h_{1}(s) d s .
\end{aligned}
$$

So (2.2) holds. The proof is completed.

Lemma 2.3 For $t, s \in[0,1]$, the functions $K_{i}(t, s)$ and $H_{i}(t, s)(i=1,2)$ defined as $(2.3)$ satisfy

$$
\begin{aligned}
& K_{1}(t, s), H_{2}(t, s) \leq \rho s(1-s)^{\alpha_{1}-1}, \quad K_{2}(t, s), H_{1}(t, s) \leq \rho s(1-s)^{\alpha_{2}-1}, \\
& K_{1}(t, s), H_{1}(t, s) \leq \rho t^{\alpha_{1}-1}, \quad K_{2}(t, s), H_{2}(t, s) \leq \rho t^{\alpha_{2}-1}, \\
& K_{1}(t, s) \geq \varrho t^{\alpha_{1}-1} s(1-s)^{\alpha_{1}-1}, \quad H_{2}(t, s) \geq \varrho t^{\alpha_{2}-1} s(1-s)^{\alpha_{1}-1}, \\
& K_{2}(t, s) \geq \varrho t^{\alpha_{2}-1} s(1-s)^{\alpha_{2}-1}, \quad H_{1}(t, s) \geq \varrho t^{\alpha_{1}-1} s(1-s)^{\alpha_{2}-1},
\end{aligned}
$$

where

$$
\begin{aligned}
& \rho=\max \left\{\begin{array}{l}
\frac{1}{\Gamma\left(\alpha_{1}-1\right)}\left(\frac{\mu_{1} \mu_{2} k_{1}}{1-\mu_{1} \mu_{2} k_{1} k_{2}} \int_{0}^{1} d A_{2}(t)+1\right), \frac{\mu_{1}}{\Gamma\left(\alpha_{2}-1\right)\left(1-\mu_{1} \mu_{2} k_{1} k_{2}\right)} \int_{0}^{1} A_{1}(t), \\
\frac{1}{\Gamma\left(\alpha_{2}-1\right)}\left(\frac{\mu_{2} \mu_{1} k_{2}}{1-\mu_{1} \mu_{2} k_{1} k_{2}} \int_{0}^{1} d A_{1}(t)+1\right), \frac{\mu_{2}}{\Gamma\left(\alpha_{1}-1\right)\left(1-\mu_{1} \mu_{2} k_{1} k_{2}\right)} \int_{0}^{1} d A_{2}(t)
\end{array}\right\}, \\
& \varrho=\min \left\{\begin{array}{l}
\frac{\mu_{1} \mu_{2} k_{1}}{\Gamma\left(\alpha_{1}\right)\left(1-\mu_{1} \mu_{2} k_{1} k_{2}\right)} \int_{0}^{1}(1-t) t^{\alpha_{1}-1} d A_{2}(t), \frac{\mu_{1}}{\Gamma\left(\alpha_{2}\right)\left(1-\mu_{1} \mu_{2} k_{1} k_{2}\right)} \int_{0}^{1}(1-t) t^{\alpha_{2}-1} d A_{1}(t), \\
\frac{\mu_{2} \mu_{1} k_{2}}{\Gamma\left(\alpha_{2}\right)\left(1-\mu_{1} \mu_{2} k_{1} k_{2}\right)} \int_{0}^{1}(1-t) t^{\alpha_{2}-1} d A_{1}(t), \frac{\mu_{2}}{\Gamma\left(\alpha_{1}\right)\left(1-\mu_{1} \mu_{2} k_{1} k_{2}\right)} \int_{0}^{1}(1-t) t^{\alpha_{1}-1} d A_{2}(t)
\end{array}\right\} .
\end{aligned}
$$


Proof By [18, Lemma 3.2], for any $t, s \in[0,1]$, we have

$$
\frac{(1-t) t^{\alpha_{i}-1} s(1-s)^{\alpha_{i}-1}}{\Gamma\left(\alpha_{i}\right)} \leq G_{i}(t, s) \leq \frac{s(1-s)^{\alpha_{i}-1}}{\Gamma\left(\alpha_{i}-1\right)}, \quad i=1,2
$$

So, by (2.3) and (2.14), we have

$$
\begin{aligned}
K_{1}(t, s) & =\frac{\mu_{1} \mu_{2} k_{1} t^{\alpha_{1}-1}}{1-\mu_{1} \mu_{2} k_{1} k_{2}} \int_{0}^{1} G_{1}(t, s) d A_{2}(t)+G_{1}(t, s) \\
& \leq \frac{\mu_{1} \mu_{2} k_{1} t^{\alpha_{1}-1}}{1-\mu_{1} \mu_{2} k_{1} k_{2}} \int_{0}^{1} \frac{s(1-s)^{\alpha_{1}-1}}{\Gamma\left(\alpha_{1}-1\right)} d A_{2}(t)+\frac{s(1-s)^{\alpha_{1}-1}}{\Gamma\left(\alpha_{1}-1\right)} \\
& \leq \frac{1}{\Gamma\left(\alpha_{1}-1\right)}\left(\frac{\mu_{1} \mu_{2} k_{1} t^{\alpha_{1}-1}}{1-\mu_{1} \mu_{2} k_{1} k_{2}} \int_{0}^{1} d A_{2}(t)+1\right) s(1-s)^{\alpha_{1}-1} \\
& \leq \frac{1}{\Gamma\left(\alpha_{1}-1\right)}\left(\frac{\mu_{1} \mu_{2} k_{1}}{1-\mu_{1} \mu_{2} k_{1} k_{2}} \int_{0}^{1} d A_{2}(t)+1\right) s(1-s)^{\alpha_{1}-1} \\
& \leq \rho s(1-s)^{\alpha_{1}-1}, \\
H_{2}(t, s) & =\frac{\mu_{2} t^{\alpha_{2}-1}}{1-\mu_{1} \mu_{2} k_{1} k_{2}} \int_{0}^{1} G_{1}(t, s) d A_{2}(t) \\
& \leq \frac{s(1-s)^{\alpha_{1}-1}}{\Gamma\left(\alpha_{1}-1\right)} \frac{\mu_{2} t^{\alpha_{2}-1}}{1-\mu_{1} \mu_{2} k_{1} k_{2}} \int_{0}^{1} d A_{2}(t) \\
& \leq\left(\frac{\mu_{2}}{\Gamma\left(\alpha_{1}-1\right)\left(1-\mu_{1} \mu_{2} k_{1} k_{2}\right)} \int_{0}^{1} d A_{2}(t)\right) s(1-s)^{\alpha_{1}-1} \\
& \leq \rho s(1-s)^{\alpha_{1}-1} .
\end{aligned}
$$

By a similar proof as (2.15) and (2.16), we also obtain

$$
K_{2}(t, s), H_{1}(t, s) \leq \rho s(1-s)^{\alpha_{2}-1}, \quad t, s \in[0,1],
$$

then we know that (2.10) holds.

By [18, Lemma 3.2], for any $t, s \in[0,1]$, we also have

$$
\frac{(1-t) t^{\alpha_{i}-1} s(1-s)^{\alpha_{i}-1}}{\Gamma\left(\alpha_{i}\right)} \leq G_{i}(t, s) \leq \frac{t^{\alpha_{i}-1}(1-t)}{\Gamma\left(\alpha_{i}-1\right)}, \quad i=1,2 .
$$

So, by (2.3) and (2.17), we have

$$
\begin{aligned}
K_{1}(t, s) & =\frac{\mu_{1} \mu_{2} k_{1} t^{\alpha_{1}-1}}{1-\mu_{1} \mu_{2} k_{1} k_{2}} \int_{0}^{1} G_{1}(t, s) d A_{2}(t)+G_{1}(t, s) \\
& \leq \frac{\mu_{1} \mu_{2} k_{1} t^{\alpha_{1}-1}}{1-\mu_{1} \mu_{2} k_{1} k_{2}} \int_{0}^{1} \frac{t^{\alpha_{1}-1}(1-t)}{\Gamma\left(\alpha_{1}-1\right)} d A_{2}(t)+\frac{t^{\alpha_{1}-1}(1-t)}{\Gamma\left(\alpha_{1}-1\right)} \\
& \leq \frac{\mu_{1} \mu_{2} k_{1} t^{\alpha_{1}-1}}{1-\mu_{1} \mu_{2} k_{1} k_{2}} \int_{0}^{1} \frac{1}{\Gamma\left(\alpha_{1}-1\right)} d A_{2}(t)+\frac{t^{\alpha_{1}-1}}{\Gamma\left(\alpha_{1}-1\right)} \\
& \leq \frac{1}{\Gamma\left(\alpha_{1}-1\right)}\left(\frac{\mu_{1} \mu_{2} k_{1}}{1-\mu_{1} \mu_{2} k_{1} k_{2}} \int_{0}^{1} d A_{2}(t)+1\right) t^{\alpha_{1}-1} \\
& \leq \rho t^{\alpha_{1}-1}
\end{aligned}
$$




$$
\begin{aligned}
H_{2}(t, s) & =\frac{\mu_{2} t^{\alpha_{2}-1}}{1-\mu_{1} \mu_{2} k_{1} k_{2}} \int_{0}^{1} G_{1}(t, s) d A_{2}(t) \\
& \leq \frac{\mu_{2} t^{\alpha_{2}-1}}{1-\mu_{1} \mu_{2} k_{1} k_{2}} \int_{0}^{1} \frac{t^{\alpha_{1}-1}(1-t)}{\Gamma\left(\alpha_{1}-1\right)} d A_{2}(t) \\
& \leq \frac{\mu_{2} t^{\alpha_{2}-1}}{1-\mu_{1} \mu_{2} k_{1} k_{2}} \int_{0}^{1} \frac{1}{\Gamma\left(\alpha_{1}-1\right)} d A_{2}(t) \leq \rho t^{\alpha_{2}-1} .
\end{aligned}
$$

By a similar proof as (2.18) and (2.19), we also obtain

$$
K_{2}(t, s) \leq \rho t^{\alpha_{2}-1}, \quad H_{1}(t, s) \leq \rho t^{\alpha_{1}-1}, \quad t \in[0,1],
$$

then we know that (2.11) holds.

On the other hand, by (2.3) and (2.14), we also have

$$
\begin{aligned}
K_{1}(t, s) & =\frac{\mu_{1} \mu_{2} k_{1} t^{\alpha_{1}-1}}{1-\mu_{1} \mu_{2} k_{1} k_{2}} \int_{0}^{1} G_{1}(t, s) d A_{2}(t)+G_{1}(t, s) \\
& \geq \frac{\mu_{1} \mu_{2} k_{1} t^{\alpha_{1}-1}}{1-\mu_{1} \mu_{2} k_{1} k_{2}} \int_{0}^{1} \frac{(1-t) t^{\alpha_{1}-1} s(1-s)^{\alpha_{1}-1}}{\Gamma\left(\alpha_{1}\right)} d A_{2}(t) \\
& \geq\left(\frac{\mu_{1} \mu_{2} k_{1}}{\Gamma\left(\alpha_{1}\right)\left(1-\mu_{1} \mu_{2} k_{1} k_{2}\right)} \int_{0}^{1}(1-t) t^{\alpha_{1}-1} d A_{2}(t)\right) t^{\alpha_{1}-1} s(1-s)^{\alpha_{1}-1} \\
& \geq \varrho t^{\alpha_{1}-1} s(1-s)^{\alpha_{1}-1}, \\
H_{2}(t, s) & =\frac{\mu_{2} t^{\alpha_{2}-1}}{1-\mu_{1} \mu_{2} k_{1} k_{2}} \int_{0}^{1} G_{1}(t, s) d A_{2}(t) \\
& \geq \frac{\mu_{2} t^{\alpha_{2}-1}}{1-\mu_{1} \mu_{2} k_{1} k_{2}} \int_{0}^{1} \frac{(1-t) t^{\alpha_{1}-1} s(1-s)^{\alpha_{1}-1}}{\Gamma\left(\alpha_{1}\right)} d A_{2}(t) \\
& \geq\left(\frac{\mu_{2}}{\Gamma\left(\alpha_{1}\right)\left(1-\mu_{1} \mu_{2} k_{1} k_{2}\right)} \int_{0}^{1}(1-t) t^{\alpha_{1}-1} d A_{2}(t)\right) t^{\alpha_{2}-1} s(1-s)^{\alpha_{1}-1} \\
& \geq \varrho t^{\alpha_{2}-1} s(1-s)^{\alpha_{1}-1} .
\end{aligned}
$$

So, we can get that (2.12) holds. By a similar proof as (2.20) and (2.21), we also obtain

$$
K_{2}(t, s) \geq \varrho t^{\alpha_{2}-1} s(1-s)^{\alpha_{2}-1}, \quad H_{1}(t, s) \geq \varrho t^{\alpha_{1}-1} s(1-s)^{\alpha_{2}-1}, \quad t \in[0,1],
$$

which implies that (2.13) holds. The proof is completed.

Remark 2.1 From Lemma 2.3, for $t, \tau, s \in[0,1]$, we have

$$
\begin{array}{ll}
K_{i}(t, s) \geq \omega t^{\alpha_{i}-1} K_{i}(\tau, s), & H_{i}(t, s) \geq \omega t^{\alpha_{i}-1} H_{i}(\tau, s), \quad i=1,2, \\
K_{1}(t, s) \geq \omega t^{\alpha_{1}-1} H_{2}(\tau, s), & H_{2}(t, s) \geq \omega t^{\alpha_{2}-1} K_{1}(\tau, s), \\
K_{2}(t, s) \geq \omega t^{\alpha_{2}-1} H_{1}(\tau, s), & H_{1}(t, s) \geq \omega t^{\alpha_{1}-1} K_{2}(\tau, s),
\end{array}
$$

where $\omega=\frac{\varrho}{\rho}, \varrho, \rho$ are defined as Lemma $2.3,0<\omega<1$.

In the rest of the paper, we always suppose that the following assumption holds: 
$\left(\mathbf{H}_{1}\right) f_{i}:(0,1) \times[0,+\infty) \times[0,+\infty) \rightarrow(-\infty,+\infty)$ is continuous and satisfies

$$
\begin{aligned}
-q_{i}(t) \leq f_{i}(t, x, y) & \leq a_{i}(t) p_{i}(t, x, y), \\
(t, x, y) \in(0,1) & \times[0,+\infty) \times[0,+\infty), i=1,2,
\end{aligned}
$$

where $a_{i}, q_{i}:(0,1) \rightarrow[0,+\infty)$ are continuous and may be singular at $t=0,1, p_{i}:[0,1] \times$ $[0,+\infty) \times[0,+\infty) \rightarrow[0,+\infty)$ is continuous and

$$
0<\int_{0}^{1} q_{i}(s) d s<+\infty, \quad 0<\int_{0}^{1} a_{i}(s) d s<+\infty, \quad i=1,2 .
$$

Lemma 2.4 Assume that $\left(\mathbf{H}_{0}\right),\left(\mathbf{H}_{1}\right)$ hold. Then the system with the coupled boundary conditions

$$
\begin{cases}D_{0^{+}}^{\alpha_{1}} \varpi_{1}(t)+\lambda_{1} q_{1}(t)=0, & \\ D_{0^{+}}^{\alpha_{2}} \varpi_{2}(t)+\lambda_{2} q_{2}(t)=0, \quad 0<t<1, & \\ \varpi_{1}(0)=\varpi_{1}^{\prime}(0)=\cdots=\varpi_{1}^{(n-2)}(0)=0, & \varpi_{1}(1)=\mu_{1} \int_{0}^{1} \varpi_{2}(s) d A_{1}(s), \\ \varpi_{2}(0)=\varpi_{2}^{\prime}(0)=\cdots=\varpi_{2}^{(n-2)}(0)=0, & \varpi_{2}(1)=\mu_{2} \int_{0}^{1} \varpi_{1}(s) d A_{2}(s)\end{cases}
$$

has a unique solution

$$
\left\{\begin{array}{l}
\varpi_{1}(t)=\lambda_{1} \int_{0}^{1} K_{1}(t, s) q_{1}(s) d s+\lambda_{2} \int_{0}^{1} H_{1}(t, s) q_{2}(s) d s \\
\varpi_{2}(t)=\lambda_{2} \int_{0}^{1} K_{2}(t, s) q_{2}(s) d s+\lambda_{1} \int_{0}^{1} H_{2}(t, s) q_{1}(s) d s
\end{array}\right.
$$

which satisfies

$$
\varpi_{i}(t) \leq \lambda_{1} \rho t^{\alpha_{i}-1} \int_{0}^{1} q_{1}(s) d s+\lambda_{2} \rho t^{\alpha_{i}-1} \int_{0}^{1} q_{2}(s) d s, \quad 0 \leq t \leq 1, i=1,2 .
$$

Proof It follows from Lemmas 2.2, 2.3 and conditions $\left(\mathbf{H}_{0}\right),\left(\mathbf{H}_{1}\right)$ that (2.22) and (2.23) hold. The proof is completed.

Next we consider the following singular nonlinear system:

$$
\left\{\begin{array}{l}
D_{0^{+}}^{\alpha_{1}} u(t)+\lambda_{1}\left(f_{1}\left(t,\left[u(t)-\varpi_{1}(t)\right]^{*},\left[v(t)-\varpi_{2}(t)\right]^{*}\right)+q_{1}(t)\right)=0, \\
D_{0^{+}}^{\alpha_{2}} v(t)+\lambda_{2}\left(f_{2}\left(t,\left[u(t)-\varpi_{1}(t)\right]^{*},\left[v(t)-\varpi_{2}(t)\right]^{*}\right)+q_{2}(t)\right)=0, \quad 0<t<1, \\
u(0)=u^{\prime}(0)=\cdots=u^{(n-2)}(0)=0, \quad u(1)=\mu_{1} \int_{0}^{1} v(s) d A_{1}(s), \\
v(0)=v^{\prime}(0)=\cdots=v^{(n-2)}(0)=0, \quad v(1)=\mu_{2} \int_{0}^{1} u(s) d A_{2}(s),
\end{array}\right.
$$

where $\lambda_{i}>0, \varpi_{i}(t)$ is defined as $(2.22),[z(t)]^{*}=\max \{z(t), 0\}(i=1,2)$.

Lemma 2.5 If $(u, v)$ is a solution of system (2.24) with $u(t)>\varpi_{1}(t), v(t)>\varpi_{2}(t)$ for any $t \in(0,1]$, then $\left(u-\varpi_{1}, v-\varpi_{2}\right)$ is a positive solution of system (1.1). 
Proof In fact, if $(u, v)$ is a positive solution of system (2.24) such that $u(t)>\varpi_{1}(t), v(t)>$ $\varpi_{2}(t)$ for any $t \in(0,1]$, then from system (2.24) and the definition of $[z(t)]^{*}$, we have

$$
\left\{\begin{array}{l}
D_{0^{+}}^{\alpha_{1}} u(t)+\lambda_{1}\left(f_{1}\left(t, u(t)-\varpi_{1}(t), v(t)-\varpi_{2}(t)\right)+q_{1}(t)\right)=0, \\
D_{0^{+}}^{\alpha_{2}} v(t)+\lambda_{2}\left(f_{2}\left(t, u(t)-\varpi_{1}(t), v(t)-\varpi_{2}(t)\right)+q_{2}(t)\right)=0, \quad 0<t<1, \\
u(0)=u^{\prime}(0)=\cdots=u^{(n-2)}(0)=0, \quad u(1)=\mu_{1} \int_{0}^{1} v(s) d A_{1}(s), \\
v(0)=v^{\prime}(0)=\cdots=v^{(n-2)}(0)=0, \quad v(1)=\mu_{2} \int_{0}^{1} u(s) d A_{2}(s) .
\end{array}\right.
$$

Let $x(t)=u(t)-\varpi_{1}(t), y(t)=v(t)-\varpi_{2}(t), t \in(0,1)$, then

$$
D_{0^{+}}^{\alpha_{1}} x(t)=D_{0^{+}}^{\alpha_{1}} u(t)-D_{0^{+}}^{\alpha_{1}} \varpi_{1}(t), \quad D_{0^{+}}^{\alpha_{2}} y(t)=D_{0^{+}}^{\alpha_{2}} v(t)-D_{0^{+}}^{\alpha_{2}} \varpi_{2}(t),
$$

which implies that

$$
D_{0^{+}}^{\alpha_{1}} u(t)=D_{0^{+}}^{\alpha_{1}} x(t)-\lambda_{1} q_{1}(t), \quad D_{0^{+}}^{\alpha_{2}} \nu(t)=D_{0^{+}}^{\alpha_{2}} y(t)-\lambda_{2} q_{2}(t) .
$$

Thus, system (2.25) becomes

$$
\left\{\begin{array}{l}
D_{0^{+}}^{\alpha_{1}} x(t)+\lambda_{1} f_{1}(t, x(t), y(t))=0, \\
D_{0^{+}}^{\alpha_{2}} y(t)+\lambda_{2} f_{2}(t, x(t), y(t))=0, \quad 0<t<1, \\
x(0)=x^{\prime}(0)=\cdots=x^{(n-2)}(0)=0, \quad x(1)=\mu_{1} \int_{0}^{1} y(s) d A_{1}(s), \\
y(0)=y^{\prime}(0)=\cdots=y^{(n-2)}(0)=0, \quad y(1)=\mu_{2} \int_{0}^{1} x(s) d A_{2}(s) .
\end{array}\right.
$$

Then, by (2.26), $\left(u-\varpi_{1}, v-\varpi_{2}\right)$ is a positive solution of system (1.1). The proof is completed.

Let $X=C[0,1] \times C[0,1]$, then $X$ is a Banach space with the norm

$$
\|(u, v)\|=\max \{\|u\|,\|v\|\}, \quad\|u\|=\max _{t \in[0,1]}|u(t)|, \quad\|v\|=\max _{t \in[0,1]}|v(t)| .
$$

Let

$$
K=\left\{(u, v) \in X: u(t) \geq \omega t^{\alpha_{1}-1}\|(u, v)\|, v(t) \geq \omega t^{\alpha_{2}-1}\|(u, v)\|, t \in[0,1]\right\},
$$

where $\omega$ is defined as Remark 2.1. It is easy to see that $K$ is a positive cone in $X$. Under the above conditions $\left(\mathbf{H}_{0}\right),\left(\mathbf{H}_{1}\right)$, for any $(u, v) \in K$, we can define an integral operator $T: K \rightarrow$ $X$ by

$$
\begin{aligned}
T(u, v)(t)= & \left(T_{1}(u, v)(t), T_{2}(u, v)(t)\right), \quad 0 \leq t \leq 1, \\
T_{i}(u, v)(t)= & \lambda_{i} \int_{0}^{1} K_{i}(t, s)\left(f_{i}\left(s,\left[u(s)-\varpi_{1}(s)\right]^{*},\left[v(s)-\varpi_{2}(s)\right]^{*}\right)+q_{i}(s)\right) d s \\
& +\lambda_{j} \int_{0}^{1} H_{i}(t, s)\left(f_{j}\left(s,\left[u(s)-\varpi_{1}(s)\right]^{*},\left[v(s)-\varpi_{2}(s)\right]^{*}\right)+q_{j}(s)\right) d s, \\
& 0 \leq t \leq 1, i=1,2, i+j=3 .
\end{aligned}
$$

We know that $(u, v)$ is a positive solutions of system (1.1) if and only if $(u, v)$ is a fixed point of $T$ in $K$. 
Lemma 2.6 Assume that $\left(\mathbf{H}_{0}\right),\left(\mathbf{H}_{1}\right)$ hold. Then $T: K \rightarrow K$ is a completely continuous operator.

Proof By a routine discussion, we know that $T: K \rightarrow X$ is well defined, so we only prove $T(K) \subseteq K$. For any $(u, v) \in K, 0 \leq t, \tau \leq 1$, by Remark 2.1, we have

$$
\begin{aligned}
T_{1}(u, v)(t)= & \lambda_{1} \int_{0}^{1} K_{1}(t, s)\left(f_{1}\left(s,\left[u(s)-\varpi_{1}(s)\right]^{*},\left[v(s)-\varpi_{2}(s)\right]^{*}\right)+q_{1}(s)\right) d s \\
& +\lambda_{2} \int_{0}^{1} H_{1}(t, s)\left(f_{2}\left(s,\left[u(s)-\varpi_{1}(s)\right]^{*},\left[v(s)-\varpi_{2}(s)\right]^{*}\right)+q_{2}(s)\right) d s \\
\geq & \lambda_{1} \int_{0}^{1} \omega t^{\alpha_{1}-1} K_{1}(\tau, s)\left(f_{1}\left(s,\left[u(s)-\varpi_{1}(s)\right]^{*},\left[v(s)-\varpi_{2}(s)\right]^{*}\right)+q_{1}(s)\right) d s \\
& +\lambda_{2} \int_{0}^{1} \omega t^{\alpha_{1}-1} H_{1}(\tau, s)\left(f_{2}\left(s,\left[u(s)-\varpi_{1}(s)\right]^{*},\left[v(s)-\varpi_{2}(s)\right]^{*}\right)+q_{2}(s)\right) d s \\
\geq & \omega t^{\alpha_{1}-1}\left(\lambda_{1} \int_{0}^{1} K_{1}(\tau, s)\left(f_{1}\left(s,\left[u(s)-\varpi_{1}(s)\right]^{*},\left[v(s)-\varpi_{2}(s)\right]^{*}\right)+q_{1}(s)\right) d s\right. \\
& \left.+\lambda_{2} \int_{0}^{1} H_{1}(\tau, s)\left(f_{2}\left(s,\left[u(s)-\varpi_{1}(s)\right]^{*},\left[v(s)-\varpi_{2}(s)\right]^{*}\right)+q_{2}(s)\right) d s\right) \\
\geq & \omega t^{\alpha_{1}-1} T_{1}(u, v)(\tau) .
\end{aligned}
$$

On the other hand,

$$
\begin{aligned}
T_{1}(u, v)(t)= & \lambda_{1} \int_{0}^{1} K_{1}(t, s)\left(f_{1}\left(s,\left[u(s)-\varpi_{1}(s)\right]^{*},\left[v(s)-\varpi_{2}(s)\right]^{*}\right)+q_{1}(s)\right) d s \\
& +\lambda_{2} \int_{0}^{1} H_{1}(t, s)\left(f_{2}\left(s,\left[u(s)-\varpi_{1}(s)\right]^{*},\left[v(s)-\varpi_{2}(s)\right]^{*}\right)+q_{2}(s)\right) d s \\
\geq & \lambda_{1} \int_{0}^{1} \omega t^{\alpha_{1}-1} H_{2}(\tau, s)\left(f_{1}\left(s,\left[u(s)-\varpi_{1}(s)\right]^{*},\left[v(s)-\varpi_{2}(s)\right]^{*}\right)+q_{1}(s)\right) d s \\
& +\lambda_{2} \int_{0}^{1} \omega t^{\alpha_{1}-1} K_{2}(\tau, s)\left(f_{2}\left(s,\left[u(s)-\varpi_{1}(s)\right]^{*},\left[v(s)-\varpi_{2}(s)\right]^{*}\right)+q_{2}(s)\right) d s \\
\geq & \omega t^{\alpha_{1}-1}\left(\lambda_{1} \int_{0}^{1} H_{2}(\tau, s)\left(f_{1}\left(s,\left[u(s)-\varpi_{1}(s)\right]^{*},\left[v(s)-\varpi_{2}(s)\right]^{*}\right)+q_{1}(s)\right) d s\right. \\
& \left.+\lambda_{2} \int_{0}^{1} K_{2}(\tau, s)\left(f_{2}\left(s,\left[u(s)-\varpi_{1}(s)\right]^{*},\left[v(s)-\varpi_{2}(s)\right]^{*}\right)+q_{2}(s)\right) d s\right) \\
\geq & \omega t^{\alpha_{1}-1} T_{2}(u, v)(\tau) .
\end{aligned}
$$

Then we have

$$
T_{1}(u, v)(t) \geq \omega t^{\alpha_{1}-1}\left\|T_{1}(u, v)\right\|, \quad T_{1}(u, v)(t) \geq \omega t^{\alpha_{1}-1}\left\|T_{2}(u, v)\right\|,
$$

i.e.,

$$
T_{1}(u, v)(t) \geq \omega t^{\alpha_{1}-1}\left\|\left(T_{1}(u, v), T_{2}(u, v)\right)\right\| .
$$


In the same way as (2.29) and (2.30), we can prove that

$$
T_{2}(u, v)(t) \geq \omega t^{\alpha_{2}-1}\left\|\left(T_{1}(u, v), T_{2}(u, v)\right)\right\| .
$$

Therefore, we have $T(K) \subseteq K$.

According to the Ascoli-Arzela theorem, we can easily get that $T: K \rightarrow K$ is completely continuous. The proof is completed.

In order to obtain the existence of the positive solutions of system (1.1), we will use the following cone compression and expansion fixed point theorem.

Lemma 2.7 [19] Let $P$ be a positive cone in a Banach space $E, \Omega_{1}$ and $\Omega_{2}$ are bounded open sets in $E, \theta \in \Omega_{1}, \bar{\Omega}_{1} \subset \Omega_{2}, A: P \cap \bar{\Omega}_{2} \backslash \Omega_{1} \rightarrow P$ is a completely continuous operator. If the following conditions are satisfied:

$$
\|A x\| \leq\|x\|, \quad \forall x \in P \cap \partial \Omega_{1}, \quad\|A x\| \geq\|x\|, \quad \forall x \in P \cap \partial \Omega_{2},
$$

or

$$
\|A x\| \geq\|x\|, \quad \forall x \in P \cap \partial \Omega_{1}, \quad\|A x\| \leq\|x\|, \quad \forall x \in P \cap \partial \Omega_{2},
$$

then $A$ has at least one fixed point in $P \cap\left(\bar{\Omega}_{2} \backslash \Omega_{1}\right)$.

\section{Main results}

Theorem 3.1 Assume that $\left(\mathbf{H}_{0}\right),\left(\mathbf{H}_{1}\right)$ hold and that for any fixed $\lambda_{1}, \lambda_{2} \in(0,+\infty)$, the following conditions are satisfied:

$\left(\mathbf{H}_{2}\right)$ There exists a constant

$$
r_{1}>\max \left\{L_{1}, L_{2}, \omega^{-1} \rho\left(\lambda_{1} \int_{0}^{1} q_{1}(s) d s+\lambda_{2} \int_{0}^{1} q_{2}(s) d s\right)\right\}
$$

such that

$$
p_{i}(t, x, y) \leq \frac{r_{1}}{L_{i}}-1, \quad(t, x, y) \in[0,1] \times\left[0, r_{1}\right] \times\left[0, r_{1}\right], i=1,2 .
$$

$\left(\mathbf{H}_{3}\right)$

$$
\begin{aligned}
& 0<l_{1}<\liminf _{x \rightarrow+\infty} \inf _{\substack{t \in[a, b] \subset(0,1) \\
y \in[0,+\infty)}} \frac{f_{1}(t, x, y)}{x} \leq+\infty, \quad \text { or } \\
& 0<l_{1}<\liminf _{y \rightarrow+\infty} \inf _{\substack{t \in[a, b] \subset(0,1) \\
x \in[0,+\infty)}} \frac{f_{1}(t, x, y)}{y} \leq+\infty,
\end{aligned}
$$

where $\omega$ is defined as Remark 2.1, $\rho$ is defined as Lemma 2.3,

$$
\begin{aligned}
& L_{i}=3\left(\lambda_{i} \rho \int_{0}^{1}\left(a_{i}(s)+q_{i}(s)\right) d s\right)^{-1}, \quad i=1,2, \\
& l_{1}=\frac{3}{2}\left(\lambda_{1} \varrho \theta^{2} \omega \int_{a}^{b} s(1-s)^{\alpha_{1}-1} d s\right)^{-1}, \quad \theta=\min _{t \in[a, b]}\left\{t^{\alpha_{1}-1}, t^{\alpha_{2}-1}\right\} .
\end{aligned}
$$


Then system (1.1) has at least one positive solution $(\bar{u}, \bar{v})$. Moreover, $(\bar{u}, \bar{v})$ satisfies $\bar{u}(t) \geq$ $\bar{l} t^{\alpha_{1}-1}, \bar{v}(t) \geq \bar{l} t^{\alpha_{2}-1}, t \in[0,1]$ for some positive constant $\bar{l}$.

Proof Let $K_{r_{1}}=\left\{(u, v) \in K:\|(u, v)\|<r_{1}\right\}$. For any $(u, v) \in \partial K_{r_{1}}, t \in[0,1]$, by the definition of $\|\cdot\|$, we know that

$$
\begin{aligned}
& {\left[u(t)-\varpi_{1}(t)\right]^{*} \leq|u(t)| \leq\|u\| \leq\|(u, v)\| \leq r_{1},} \\
& {\left[v(t)-\varpi_{2}(t)\right]^{*} \leq|v(t)| \leq\|v\| \leq\|(u, v)\| \leq r_{1} .}
\end{aligned}
$$

So, for any $(u, v) \in \partial K_{r_{1}}$, by condition $\left(\mathbf{H}_{2}\right)$ and Lemma 2.3, we have

$$
\begin{aligned}
\left\|T_{1}(u, v)\right\|= & \max _{t \in[0,1]} \mid \lambda_{1} \int_{0}^{1} K_{1}(t, s)\left(f_{1}\left(s,\left[u(s)-\varpi_{1}(s)\right]^{*},\left[v(s)-\varpi_{2}(s)\right]^{*}\right)+q_{1}(s)\right) d s \\
& +\lambda_{2} \int_{0}^{1} H_{1}(t, s)\left(f_{2}\left(s,\left[u(s)-\varpi_{1}(s)\right]^{*},\left[v(s)-\varpi_{2}(s)\right]^{*}\right)+q_{2}(s)\right) d s \mid \\
\leq & \max _{t \in[0,1]} \mid \lambda_{1} \int_{0}^{1} \rho t^{\alpha_{1}-1}\left(a_{1}(s) p_{1}\left(s,\left[u(s)-\varpi_{1}(s)\right]^{*},\left[v(s)-\varpi_{2}(s)\right]^{*}\right)+q_{1}(s)\right) d s \\
& +\lambda_{2} \int_{0}^{1} \rho t^{\alpha_{1}-1}\left(a_{2}(s) p_{2}\left(s,\left[u(s)-\varpi_{1}(s)\right]^{*},\left[v(s)-\varpi_{2}(s)\right]^{*}\right)+q_{2}(s)\right) d s \mid \\
\leq & \lambda_{1} \rho \int_{0}^{1}\left(a_{1}(s)\left(\frac{r_{1}}{L_{1}}-1\right)+q_{1}(s)\right) d s+\lambda_{2} \rho \int_{0}^{1}\left(a_{2}(s)\left(\frac{r_{1}}{L_{2}}-1\right)+q_{2}(s)\right) d s \\
\leq & \left(\frac{r_{1}}{L_{1}}-1+1\right) \lambda_{1} \rho \int_{0}^{1}\left(a_{1}(s)+q_{1}(s)\right) d s \\
& +\left(\frac{r_{1}}{L_{2}}-1+1\right) \lambda_{2} \rho \int_{0}^{1}\left(a_{2}(s)+q_{2}(s)\right) d s \\
= & \frac{2 r_{1}}{3}<r_{1}=\|(u, v)\| .
\end{aligned}
$$

Similarly as (3.1), for any $(u, v) \in \partial K_{r_{1}}$, by condition $\left(\mathbf{H}_{2}\right)$, we also have

$$
\left\|T_{2}(u, v)\right\|<r_{1}=\|(u, v)\| .
$$

Consequently, we have

$$
\|T(u, v)\|=\max \left\{\left\|T_{1}(u, v)\right\|,\left\|T_{2}(u, v)\right\|\right\}<r_{1}=\|(u, v)\| \quad \text { for any }(u, v) \in \partial K_{r_{1}} .
$$

On the other hand, by the first inequality in $\left(\mathbf{H}_{3}\right)$, there exists $\varepsilon_{0}>0$ such that $l_{1}+\varepsilon_{0}>0$, and also there exists $r_{0}>0$ such that

$$
\left|f_{1}(t, x, y)\right| \geq\left(l_{1}+\varepsilon_{0}\right) x, \quad x \geq r_{0}, y \geq 0, t \in[a, b] .
$$


Choose $r_{2}=\max \left\{3 r_{1}, \frac{3 r_{0}}{2 \omega \theta}\right\}$. Let $K_{r_{2}}=\left\{(u, v) \in K:\|(u, v)\|<r_{2}\right\}$. For any $(u, v) \in \partial K_{r_{2}}$, by the definition of $\|\cdot\|$ and (2.23), we have

$$
\begin{aligned}
u(t)-\varpi_{1}(t) & \geq \omega t^{\alpha_{1}-1} r_{2}-\left(\lambda_{1} \rho t^{\alpha_{1}-1} \int_{0}^{1} q_{1}(s) d s+\lambda_{2} \rho t^{\alpha_{1}-1} \int_{0}^{1} q_{2}(s) d s\right) \\
& =t^{\alpha_{1}-1}\left(\omega r_{2}-\left(\lambda \rho \int_{0}^{1} q_{1}(s) d s+\lambda_{2} \rho \int_{0}^{1} q_{2}(s) d s\right)\right) \\
& \geq \theta\left(\omega r_{2}-\rho\left(\lambda_{1} \int_{0}^{1} q_{1}(s) d s+\lambda_{2} \int_{0}^{1} q_{2}(s) d s\right)\right) \\
& \geq \omega \theta\left(r_{2}-r_{1}\right) \geq \frac{2 \omega \theta r_{2}}{3} \geq r_{0}, \quad t \in[a, b], \\
v(t)-\varpi_{2}(t) & \geq \omega t^{\alpha_{2}-1} r_{2}-\left(\lambda_{1} \rho t^{\alpha_{2}-1} \int_{0}^{1} q_{1}(s) d s+\lambda_{2} \rho t^{\alpha_{2}-1} \int_{0}^{1} q_{2}(s) d s\right) \\
& =t^{\alpha_{2}-1}\left(\omega r_{2}-\left(\lambda_{1} \rho \int_{0}^{1} q_{1}(s) d s+\lambda_{2} \rho \int_{0}^{1} q_{2}(s) d s\right)\right) \\
& \geq \theta\left(\omega r_{2}-\rho\left(\lambda_{1} \int_{0}^{1} q_{1}(s) d s+\lambda_{2} \int_{0}^{1} q_{2}(s) d s\right)\right) \\
& \geq \omega \theta\left(r_{2}-r_{1}\right) \geq \frac{2 \omega \theta r_{2}}{3} \geq r_{0}>0, \quad t \in[a, b] .
\end{aligned}
$$

Thus, for any $(u, v) \in \partial K_{r_{2}}$, by (3.3)-(3.5), we have

$$
f_{1}\left(t,\left[u(t)-\varpi_{1}(t)\right]^{*},\left[v(t)-\varpi_{2}(t)\right]^{*}\right) \geq\left(l_{1}+\varepsilon_{0}\right)\left[u(t)-\varpi_{1}(t)\right]^{*}, \quad t \in[a, b] .
$$

Hence, for any $(u, v) \in \partial K_{r_{2}}$, by (3.6) and Lemma 2.3, we conclude that

$$
\begin{aligned}
\left\|T_{1}(u, v)\right\|= & \max _{t \in[0,1]} \mid \lambda_{1} \int_{0}^{1} K_{1}(t, s)\left(f_{1}\left(s,\left[u(s)-\varpi_{1}(s)\right]^{*},\left[v(s)-\varpi_{2}(s)\right]^{*}\right)+q_{1}(s)\right) d s \\
& +\lambda_{2} \int_{0}^{1} H_{1}(t, s)\left(f_{2}\left(s,\left[u(s)-\varpi_{1}(s)\right]^{*},\left[v(s)-\varpi_{2}(s)\right]^{*}\right)+q_{2}(s)\right) d s \mid \\
\geq & \max _{t \in[0,1]} \lambda_{1} \int_{0}^{1} K_{1}(t, s)\left(f_{1}\left(s,\left[u(s)-\varpi_{1}(s)\right]^{*},\left[v(s)-\varpi_{2}(s)\right]^{*}\right)+q_{1}(s)\right) d s \\
\geq & \max _{t \in[0,1]} \lambda_{1} \int_{a}^{b} K_{1}(t, s) f_{1}\left(s,\left[u(s)-\varpi_{1}(s)\right]^{*},\left[v(s)-\varpi_{2}(s)\right]^{*}\right) d s \\
\geq & \min _{t \in[a, b]} \lambda_{1} \int_{a}^{b} \varrho t^{\alpha_{1}-1} s(1-s)^{\alpha_{1}-1}\left(l_{1}+\varepsilon_{0}\right)\left[u(s)-\varpi_{1}(s)\right]^{*} d s \\
\geq & \frac{2 \lambda_{1} \varrho \theta^{2}\left(l_{1}+\varepsilon_{0}\right) \omega r_{2}}{3} \int_{a}^{b} s(1-s)^{\alpha_{1}-1} d s \\
\geq & r_{2}=\|(u, v)\| .
\end{aligned}
$$

Consequently,

$$
\|T(u, v)\|=\max \left\{\left\|T_{1}(u, v)\right\|,\left\|T_{2}(u, v)\right\|\right\} \geq r_{2}=\|(u, v)\| \quad \text { for any }(u, v) \in \partial K_{r_{2}} .
$$

Obviously, by the second inequality in $\left(\mathbf{H}_{3}\right),(3.7)$ is still valid. 
It follows from the above discussion, (3.2), (3.7), Lemmas 2.6 and 2.7, that for any fixed $\lambda_{1}, \lambda_{2} \in(0,+\infty), T$ has a fixed point $(u, v) \in \bar{K}_{r_{2}} \backslash K_{r_{1}}$ and $r_{1} \leq\|(u, v)\| \leq r_{2}$. Since $\|(u, v)\| \geq$ $r_{1}$, we have

$$
\begin{aligned}
u(t)-\varpi_{1}(t) & \geq u(t)-\left(\lambda_{1} \rho t^{\alpha_{1}-1} \int_{0}^{1} q_{1}(s) d s+\lambda_{2} \rho t^{\alpha_{1}-1} \int_{0}^{1} q_{2}(s) d s\right) \\
& \geq \omega t^{\alpha_{1}-1} r_{1}-\left(\lambda_{1} \rho t^{\alpha_{1}-1} \int_{0}^{1} q_{1}(s) d s+\lambda_{2} \rho t^{\alpha_{1}-1} \int_{0}^{1} q_{2}(s) d s\right) \\
& \geq t^{\alpha_{1}-1}\left(\omega r_{1}-\lambda_{1} \rho \int_{0}^{1} q_{1}(s) d s-\lambda_{2} \rho \int_{0}^{1} q_{2}(s) d s\right)>0, \quad t \in(0,1] \\
v(t)-\varpi_{2}(t) & \geq v(t)-\left(\lambda_{1} \rho t^{\alpha_{2}-1} \int_{0}^{1} q_{1}(s) d s+\lambda_{2} \rho t^{\alpha_{2}-1} \int_{0}^{1} q_{2}(s) d s\right) \\
& \geq \omega t^{\alpha_{2}-1} r_{1}-\left(\lambda_{1} \rho t^{\alpha_{2}-1} \int_{0}^{1} q_{1}(s) d s+\lambda_{2} \rho t^{\alpha_{2}-1} \int_{0}^{1} q_{2}(s) d s\right) \\
& \geq t^{\alpha_{2}-1}\left(\omega r_{1}-\lambda_{1} \rho \int_{0}^{1} q_{1}(s) d s-\lambda_{2} \rho \int_{0}^{1} q_{2}(s) d s\right)>0, \quad t \in(0,1] .
\end{aligned}
$$

Let $\bar{l}=\omega r_{1}-\lambda_{1} \rho \int_{0}^{1} q_{1}(s) d s-\lambda_{2} \rho \int_{0}^{1} q_{2}(s) d s, \bar{u}(t)=u(t)-\varpi_{1}(t), \bar{v}(t)=v(t)-\varpi_{2}(t)$, then we have

$$
\bar{u}(t) \geq \bar{l} t^{\alpha_{1}-1}>0, \quad \bar{v}(t) \geq \bar{l} t^{\alpha_{2}-1}>0, \quad t \in(0,1]
$$

By Lemma 2.5, we know that for any fixed $\lambda_{1}, \lambda_{2} \in(0,+\infty)$, system (1.1) has at least one positive solution $(\bar{u}, \bar{v})$; moreover, $(\bar{u}, \bar{v})$ satisfies $\bar{u}(t) \geq \bar{l} t^{\alpha_{1}-1}, \bar{v}(t) \geq \bar{l} t^{\alpha_{2}-1}, t \in[0,1]$. The proof is completed.

Remark 3.1 From the proof of Theorem 3.1, we know that the conclusion of Theorem 3.1 is valid if condition $\left(\mathbf{H}_{3}\right)$ is replaced by

$$
\begin{aligned}
& 0<l_{2}<\liminf _{x \rightarrow+\infty} \inf _{\substack{t \in[a, b] \subset(0,1) \\
y \in[0,+\infty)}} \frac{f_{2}(t, x, y)}{x} \leq+\infty, \quad \text { or } \\
& 0<l_{2}<\liminf _{y \rightarrow+\infty} \inf _{\substack{t \in[a, b] \subset(0,1) \\
x \in[0,+\infty)}} \frac{f_{2}(t, x, y)}{y} \leq+\infty,
\end{aligned}
$$

where

$$
l_{2}=\frac{3}{2}\left(\lambda_{2} \varrho \theta^{2} \omega \int_{a}^{b} s(1-s)^{\alpha_{2}-1} d s\right)^{-1} .
$$

Theorem 3.2 Assume that $\left(\mathbf{H}_{0}\right),\left(\mathbf{H}_{1}\right)$ hold and that for any fixed $\lambda_{1}, \lambda_{2} \in(0,+\infty)$, the following conditions are satisfied:

$\left(\mathbf{H}_{4}\right)$ There exists a constant

$$
R_{1}>\omega^{-1} \rho\left(\lambda_{1} \int_{0}^{1} q_{1}(s) d s+\lambda_{2} \int_{0}^{1} q_{2}(s) d s\right)
$$


such that

$$
f_{1}(t, x, y) \geq \frac{R_{1}}{l_{1}}, \quad(t, x, y) \in[a, b] \times\left[0, R_{1}\right] \times\left[0, R_{1}\right]
$$

$\left(\mathbf{H}_{5}\right)$

$$
0 \leq \limsup _{x \rightarrow+\infty} \sup _{\substack{t \in[0,1] \\ y \in[0,+\infty)}} \frac{p_{i}(t, x, y)}{x}<L_{i}, \quad \text { or } \quad 0 \leq \limsup _{y \rightarrow+\infty} \sup _{\substack{t \in[0,1] \\ x \in[0,+\infty)}} \frac{p_{i}(t, x, y)}{y}<L_{i}, \quad i=1,2 \text {, }
$$

where $[a, b] \subset(0,1), L_{i}(i=1,2), l_{1}$ are defined in Theorem 3.1. Then system (1.1) has at least one positive solution $\left(\bar{u}^{0}, \bar{v}^{0}\right)$. Moreover, $\left(\bar{u}^{0}, \bar{v}^{0}\right)$ satisfies $\bar{u}^{0}(t) \geq \bar{l}^{0} t^{\alpha_{1}-1}, \bar{v}^{0}(t) \geq \bar{l}^{0} t^{\alpha_{2}-1}$, $t \in[0,1]$ for some positive constant $\bar{l}^{0}$.

The proof of Theorem 3.2 is similar to that of Theorem 3.1, and so we omit it.

Remark 3.2 The conclusion of Theorem 3.2 is valid if inequality (3.8) in condition $\left(\mathbf{H}_{6}\right)$ is replaced by

$$
f_{2}(t, x, y) \geq \frac{R_{1}}{l_{2}}, \quad(t, x, y) \in[a, b] \times\left[0, R_{1}\right] \times\left[0, R_{1}\right]
$$

where $l_{2}$ is defined in Remark 3.1.

Theorem 3.3 Assume that $\left(\mathbf{H}_{0}\right),\left(\mathbf{H}_{1}\right)$ hold and that the following is satisfied:

$$
\lim _{x \rightarrow+\infty} \inf _{\substack{t \in[c, d] \subset(0,1) \\
y \in[0,+\infty)}} \frac{f_{1}(t, x, y)}{x}=+\infty, \quad \text { or } \quad \lim _{\substack { y \rightarrow+\infty \\
\begin{subarray}{c}{t \in[c, d] \subset(0,1) \\
x \in[0,+\infty){ y \rightarrow + \infty \\
\begin{subarray} { c } { t \in [ c , d ] \subset ( 0 , 1 ) \\
x \in [ 0 , + \infty ) } }\end{subarray}} \frac{f_{1}(t, x, y)}{y}=+\infty .
$$

Then there exist $\bar{\lambda}_{1}>0, \bar{\lambda}_{2}>0$ such that system (1.1) has at least one positive solution $\left(\bar{u}^{\prime}, \bar{v}^{\prime}\right)$ provided $\lambda_{1} \in\left(0, \bar{\lambda}_{1}\right), \lambda_{2} \in\left(0, \bar{\lambda}_{2}\right)$. Moreover, $\left(\bar{u}^{\prime}, \bar{v}^{\prime}\right)$ satisfies $\bar{u}^{\prime}(t) \geq \bar{l}^{\prime} t^{\alpha_{1}-1}, \bar{v}^{\prime}(t) \geq \bar{l}^{\prime} t^{\alpha_{2}-1}$, $t \in[0,1]$ for some positive constant $\bar{l}^{\prime}$.

Proof Choose $R>\omega^{-1} \rho\left(\int_{0}^{1} q_{1}(s) d s+\int_{0}^{1} q_{2}(s) d s\right)$. Let

$$
\bar{\lambda}_{i}=\min \left\{1, \frac{R}{2 \rho \int_{0}^{1}\left(a_{i}(s) S_{i, R}+q_{i}(s)\right) d s}\right\}, \quad i=1,2,
$$

where $\omega$ is defined as Remark 2.1, $\rho$ is defined as Lemma 2.3, $S_{i, R}:=\sup \left\{p_{i}(t, x, y): 0 \leq t \leq\right.$ $1,0 \leq x, y \leq R\}(i=1,2)$.

Let $K_{R}=\{(u, v) \in K:\|(u, v)\|<R\}$. For any $(u, v) \in \partial K_{R}, t \in[0,1]$, by the definition of $\|\cdot\|$, we know that

$$
\begin{gathered}
{\left[u(t)-\varpi_{1}(t)\right]^{*} \leq|u(t)| \leq\|u\| \leq\|(u, v)\| \leq R,} \\
{\left[v(t)-\varpi_{2}(t)\right]^{*} \leq|v(t)| \leq\|v\| \leq\|(u, v)\| \leq R .}
\end{gathered}
$$


So, for $\lambda_{i} \in\left(0, \bar{\lambda}_{i}\right),(u, v) \in \partial K_{R}$, by Lemma 2.3 , we have

$$
\begin{aligned}
\left\|T_{1}(u, v)\right\|= & \max _{t \in[0,1]} \mid \lambda_{1} \int_{0}^{1} K_{1}(t, s)\left(f_{1}\left(s,\left[u(s)-\varpi_{1}(s)\right]^{*},\left[v(s)-\varpi_{2}(s)\right]^{*}\right)+q_{1}(s)\right) d s \\
& +\lambda_{2} \int_{0}^{1} H_{1}(t, s)\left(f_{2}\left(s,\left[u(s)-\varpi_{1}(s)\right]^{*},\left[v(s)-\varpi_{2}(s)\right]^{*}\right)+q_{2}(s)\right) d s \mid \\
\leq & \max _{t \in[0,1]} \mid \lambda_{1} \int_{0}^{1} \rho t^{\alpha_{1}-1}\left(a_{1}(s) p_{1}\left(s,\left[u(s)-\varpi_{1}(s)\right]^{*},\left[v(s)-\varpi_{2}(s)\right]^{*}\right)+q_{1}(s)\right) d s \\
& +\lambda_{2} \int_{0}^{1} \rho t^{\alpha_{1}-1}\left(a_{2}(s) p_{2}\left(s,\left[u(s)-\varpi_{1}(s)\right]^{*},\left[v(s)-\varpi_{2}(s)\right]^{*}\right)+q_{2}(s)\right) d s \mid \\
\leq & \lambda_{1} \rho \int_{0}^{1}\left(a_{1}(s) S_{1, R}+q_{1}(s)\right) d s+\lambda_{2} \rho \int_{0}^{1}\left(a_{2}(s) S_{2, R}+q_{2}(s)\right) d s \\
\leq & R=\|(u, v)\| .
\end{aligned}
$$

Similarly as (3.9), for any $(u, v) \in \partial K_{R}$, by condition $\left(\mathbf{H}_{2}\right)$, we also have

$$
\left\|T_{2}(u, v)\right\| \leq R=\|(u, v)\| .
$$

Consequently, we have

$$
\|T(u, v)\|=\max \left\{\left\|T_{1}(u, v)\right\|,\left\|T_{2}(u, v)\right\|\right\} \leq R=\|(u, v)\| \quad \text { for any }(u, v) \in \partial K_{R} .
$$

On the other hand, by the first inequality in $\left(\mathbf{H}_{6}\right)$, choose $M_{1}$ such that

$$
\lambda_{1} \varrho \theta^{\prime 2} M_{1} \omega \int_{c}^{d} s(1-s)^{\alpha_{1}-1} d s>2, \quad \theta^{\prime}=\min _{t \in[c, d]}\left\{t^{\alpha_{1}-1}, t^{\alpha_{2}-1}\right\}
$$

where $\omega$ is defined as Remark 2.1, $\varrho$ is defined as Lemma 2.3. Then there exists $N^{*}>0$ such that

$$
f_{1}(t, x, y) \geq M_{1} x, \quad x \geq N^{*}, y \geq 0, t \in[c, d] .
$$

Let

$$
K_{R^{\prime}}=\left\{(x, y) \in K:\|(x, y)\|<R^{\prime}\right\}, \quad R^{\prime}>\max \left\{2 R, \frac{2 N^{*}}{\omega \theta^{\prime}}\right\} .
$$

For any $(x, y) \in \partial K_{R^{\prime}}$, by (2.23), we have

$$
\begin{aligned}
u(t)-\varpi_{1}(t) & \geq \omega t^{\alpha_{1}-1} R^{\prime}-\left(\lambda_{1} \rho t^{\alpha_{1}-1} \int_{0}^{1} q_{1}(s) d s+\lambda_{2} \rho t^{\alpha_{1}-1} \int_{0}^{1} q_{2}(s) d s\right) \\
& =t^{\alpha_{1}-1}\left(\omega R^{\prime}-\rho\left(\lambda_{1} \int_{0}^{1} q_{1}(s) d s+\lambda_{2} \int_{0}^{1} q_{2}(s) d s\right)\right) \\
& \geq \theta^{\prime}\left(\omega R^{\prime}-\rho\left(\int_{0}^{1} q_{1}(s) d s+\int_{0}^{1} q_{2}(s) d s\right)\right) \\
& \geq \omega \theta^{\prime}\left(R^{\prime}-R\right) \geq \frac{\omega \theta^{\prime} R^{\prime}}{2} \geq N^{*}, \quad t \in[c, d],
\end{aligned}
$$




$$
\begin{aligned}
v(t)-\varpi_{2}(t) & \geq \omega t^{\alpha_{2}-1} R^{\prime}-\left(\lambda_{1} \rho t^{\alpha_{2}-1} \int_{0}^{1} q_{1}(s) d s+\lambda_{2} \rho t^{\alpha_{2}-1} \int_{0}^{1} q_{2}(s) d s\right) \\
& =t^{\alpha_{2}-1}\left(\omega R^{\prime}-\left(\lambda_{1} \rho \int_{0}^{1} q_{1}(s) d s+\lambda_{2} \rho \int_{0}^{1} q_{2}(s) d s\right)\right) \\
& \geq \theta^{\prime}\left(\omega R^{\prime}-\rho\left(\int_{0}^{1} q_{1}(s) d s+\int_{0}^{1} q_{2}(s) d s\right)\right) \\
& \geq \omega \theta^{\prime}\left(R^{\prime}-R\right) \geq \frac{\omega \theta^{\prime} R^{\prime}}{2} \geq N^{*}>0, \quad t \in[c, d] .
\end{aligned}
$$

Thus, for any $(u, v) \in \partial K_{R^{\prime}}$, by (3.11)-(3.13), we have

$$
f_{1}\left(t,\left[u(t)-\varpi_{1}(t)\right]^{*},\left[v(t)-\varpi_{2}(t)\right]^{*}\right) \geq M_{1}\left[u(t)-\varpi_{1}(t)\right]^{*}, \quad t \in[a, b] .
$$

Hence, for any $(u, v) \in \partial K_{R^{\prime}}$, by (3.14) and Lemma 2.3, we have

$$
\begin{aligned}
\left\|T_{1}(u, v)\right\|= & \max _{t \in[0,1]} \mid \lambda_{1} \int_{0}^{1} K_{1}(t, s)\left(f_{1}\left(s,\left[u(s)-\varpi_{1}(s)\right]^{*},\left[v(s)-\varpi_{2}(s)\right]^{*}\right)+q_{1}(s)\right) d s \\
& +\lambda_{2} \int_{0}^{1} H_{1}(t, s)\left(f_{2}\left(s,\left[u(s)-\varpi_{1}(s)\right]^{*},\left[v(s)-\varpi_{2}(s)\right]^{*}\right)+q_{2}(s)\right) d s \mid \\
\geq & \max _{t \in[0,1]} \lambda_{1} \int_{0}^{1} K_{1}(t, s)\left(f_{1}\left(s,\left[u(s)-\varpi_{1}(s)\right]^{*},\left[v(s)-\varpi_{2}(s)\right]^{*}\right)+q_{1}(s)\right) d s \\
\geq & \max _{t \in[0,1]} \lambda_{1} \int_{c}^{d} K_{1}(t, s) f_{1}\left(s,\left[u(s)-\varpi_{1}(s)\right]^{*},\left[v(s)-\varpi_{2}(s)\right]^{*}\right) d s \\
\geq & \min _{t \in[c, d]} \lambda_{1} \int_{c}^{d} \varrho t^{\alpha_{1}-1} s(1-s)^{\alpha_{1}-1} M_{1}\left[u(s)-\varpi_{1}(s)\right]^{*} d s \\
\geq & \frac{\lambda_{1} \varrho \theta^{\prime 2} M_{1} \omega R^{\prime}}{2} \int_{c}^{d} s(1-s)^{\alpha_{1}-1} d s \\
\geq & R^{\prime}=\|(u, v)\| .
\end{aligned}
$$

Consequently,

$$
\|T(u, v)\|=\max \left\{\left\|T_{1}(u, v)\right\|,\left\|T_{2}(u, v)\right\|\right\} \geq R^{\prime}=\|(u, v)\| \quad \text { for any }(u, v) \in \partial K_{R^{\prime}} .
$$

Obviously, by the second inequality in $\left(\mathbf{H}_{6}\right),(3.15)$ is still valid.

It follows from the above discussion, (3.10), (3.15), Lemmas 2.6 and 2.7, that for any $\lambda_{1} \in\left(0, \bar{\lambda}_{1}\right), \lambda_{2} \in\left(0, \bar{\lambda}_{2}\right), T$ has a fixed point $(u, v) \in \bar{K}_{R^{\prime}} \backslash K_{R}$ and $R \leq\|(u, v)\| \leq R^{\prime}$. Since $\|(u, v)\| \geq R$, we have

$$
\begin{aligned}
u(t)-\varpi_{1}(t) & \geq u(t)-\left(\lambda_{1} \rho t^{\alpha_{1}-1} \int_{0}^{1} q_{1}(s) d s+\lambda_{2} \rho t^{\alpha_{1}-1} \int_{0}^{1} q_{2}(s) d s\right) \\
& \geq \omega t^{\alpha_{1}-1} R-\left(\lambda_{1} \rho t^{\alpha_{1}-1} \int_{0}^{1} q_{1}(s) d s+\lambda_{2} \rho t^{\alpha_{1}-1} \int_{0}^{1} q_{2}(s) d s\right) \\
& \geq t^{\alpha_{1}-1}\left(\omega R-\rho \int_{0}^{1} q_{1}(s) d s-\rho \int_{0}^{1} q_{2}(s) d s\right)>0, \quad t \in(0,1]
\end{aligned}
$$




$$
\begin{aligned}
v(t)-\varpi_{2}(t) & \geq v(t)-\left(\lambda_{1} \rho t^{\alpha_{2}-1} \int_{0}^{1} q_{1}(s) d s+\lambda_{2} \rho t^{\alpha_{2}-1} \int_{0}^{1} q_{2}(s) d s\right) \\
& \geq \omega t^{\alpha_{2}-1} R-\left(\lambda_{1} \rho t^{\alpha_{2}-1} \int_{0}^{1} q_{1}(s) d s+\lambda_{2} \rho t^{\alpha_{2}-1} \int_{0}^{1} q_{2}(s) d s\right) \\
& \geq t^{\alpha_{2}-1}\left(\omega R-\rho \int_{0}^{1} q_{1}(s) d s-\rho \int_{0}^{1} q_{2}(s) d s\right)>0, \quad t \in(0,1] .
\end{aligned}
$$

Let $\bar{l}^{\prime}=\omega R-\rho \int_{0}^{1} q_{1}(s) d s-\rho \int_{0}^{1} q_{2}(s) d s, \bar{u}^{\prime}(t)=u(t)-\varpi_{1}(t), \bar{v}^{\prime}(t)=v(t)-\varpi_{2}(t)$, then we have

$$
\bar{u}^{\prime}(t) \geq \bar{l}^{\prime} t^{\alpha_{1}-1}>0, \quad \bar{v}^{\prime}(t) \geq \bar{l}^{\prime} t^{\alpha_{2}-1}>0, \quad t \in[0,1] .
$$

By Lemma 2.5 we know that for any $\lambda_{1} \in\left(0, \bar{\lambda}_{1}\right), \lambda_{2} \in\left(0, \bar{\lambda}_{2}\right)$, system (1.1) has at least one positive solution $\left(\bar{u}^{\prime}, \bar{v}^{\prime}\right)$; moreover, $\left(\bar{u}^{\prime}, \bar{v}^{\prime}\right)$ satisfies $\bar{u}^{\prime}(t) \geq \bar{l}^{\prime} t^{\alpha_{1}-1}, \bar{v}^{\prime}(t) \geq \bar{l}^{\prime} t^{\alpha_{2}-1}, t \in[0,1]$. The proof is completed.

Remark 3.3 From the proof of Theorem 3.3, we know that the conclusion of Theorem 3.3 is valid if condition $\left(\mathbf{H}_{6}\right)$ is replaced by

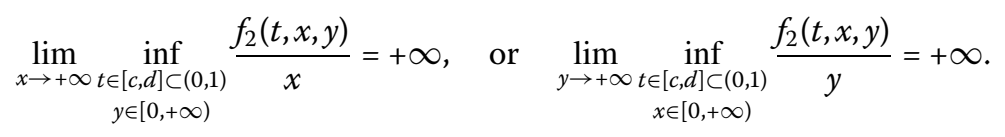

Theorem 3.4 Assume that $\left(\mathbf{H}_{0}\right),\left(\mathbf{H}_{1}\right)$ hold and that the following condition is satisfied:

$\left(\mathbf{H}_{7}\right)$

$$
\limsup _{x \rightarrow+\infty} \sup _{\substack{t \in[0,1] \\ y \in[0,+\infty)}} \frac{p_{i}(t, x, y)}{x}=0, \quad i=1,2
$$

and

$$
\liminf _{x \rightarrow+\infty} \inf _{\substack{t \in[\tilde{a}, \tilde{b}] \subset(0,1) \\
y \in[0,+\infty)}} f_{1}(t, x, y)>\wedge, \quad \text { or } \quad \liminf _{\substack { y \rightarrow+\infty \\
\begin{subarray}{c}{t \in[\tilde{a}, \tilde{b}] \subset(0,1) \\
x \in[0,+\infty){ y \rightarrow + \infty \\
\begin{subarray} { c } { t \in [ \tilde { a } , \tilde { b } ] \subset ( 0 , 1 ) \\
x \in [ 0 , + \infty ) } }\end{subarray}} f_{1}(t, x, y)>\wedge
$$

where $\wedge=\frac{4 \int_{0}^{1}\left(q_{1}(s)+q_{2}(s)\right) d s}{\omega^{2} \widetilde{\theta} \int_{\tilde{a}}^{\hat{b}} s(1-s)^{\alpha} 0^{-1} d s}, \alpha_{0}=\max \left\{\alpha_{1}, \alpha_{2}\right\}$. Then there exist $\tilde{\lambda}_{1}, \tilde{\lambda}_{2}>0$ such that system (1.1) has at least one positive solution $(\tilde{u}, \tilde{v})$ provided $\lambda_{1} \in\left(\tilde{\lambda}_{1},+\infty\right), \lambda_{2} \in\left(\tilde{\lambda}_{2},+\infty\right)$. Moreover, $(\tilde{u}, \tilde{v})$ satisfies $\tilde{u}(t) \geq \tilde{l} t^{\alpha_{1}-1}, \tilde{v}(t) \geq \tilde{l} t^{\alpha_{2}-1}, t \in[0,1]$ for some positive constant $\tilde{l}$.

Proof It follows from

$$
\liminf _{x \rightarrow+\infty} \inf _{\substack{t \in[\tilde{a}, \tilde{b}] \subset(0,1) \\ y \in[0,+\infty)}} f_{1}(t, x, y)>\wedge
$$

of $\left(\mathbf{H}_{7}\right)$, there exists $\widetilde{N}>0$ such that

$$
f_{1}(t, x, y) \geq \wedge, \quad x \geq \tilde{N}, y \geq 0, t \in[\tilde{a}, \tilde{b}] .
$$


Select

$$
\tilde{\lambda}_{i}=\frac{\tilde{N}}{2 \rho \tilde{\theta} \int_{0}^{1} q_{i}(s) d s}, \quad i=1,2 .
$$

In proving the theorem, we assume

$$
R_{1}=\max \left\{\left\{\lambda_{1}+\lambda_{2}, 2 \lambda_{1}, 2 \lambda_{2}\right\} 2 \omega^{-1} \rho \int_{0}^{1}\left(q_{1}(s)+q_{2}(s)\right) d s\right\}
$$

and

$$
K_{R_{1}}=\left\{(u, v) \in K:\|(u, v)\|<R_{1}\right\},
$$

where $\widetilde{\theta}=\min _{t \in[\widetilde{a}, \widetilde{b}]}\left\{t^{\alpha_{1}-1}, t^{\alpha_{2}-1}\right\}$. For any $(u, v) \in \partial K_{R_{1}}$, by (2.23), we have

$$
\begin{aligned}
u(t)-\varpi_{1}(t) & \geq \omega t^{\alpha_{1}-1} R_{1}-\left(\lambda_{1} \rho t^{\alpha_{1}-1} \int_{0}^{1} q_{1}(s) d s+\lambda_{2} \rho t^{\alpha_{1}-1} \int_{0}^{1} q_{2}(s) d s\right) \\
& =t^{\alpha_{1}-1}\left(\omega R_{1}-\left(\lambda_{1} \rho \int_{0}^{1} q_{1}(s) d s+\lambda_{2} \rho \int_{0}^{1} q_{2}(s) d s\right)\right) \\
& \geq \widetilde{\theta}\left(\omega R_{1}-\left(\lambda_{1} \rho \int_{0}^{1} q_{1}(s) d s+\lambda_{2} \rho \int_{0}^{1} q_{2}(s) d s\right)\right) \\
& \geq \lambda_{1} \rho \tilde{\theta} \int_{0}^{1} q_{1}(s) d s+\lambda_{2} \rho \tilde{\theta} \int_{0}^{1} q_{2}(s) d s \geq \tilde{N}, \quad t \in[\tilde{a}, \tilde{b}], \\
v(t)-\varpi_{2}(t) & \geq \omega t^{\alpha_{2}-1} R_{1}-\left(\lambda_{1} \rho t^{\alpha_{2}-1} \int_{0}^{1} q_{1}(s) d s+\lambda_{2} \rho t^{\alpha_{2}-1} \int_{0}^{1} q_{2}(s) d s\right) \\
& =t^{\alpha_{2}-1}\left(\omega R_{1}-\left(\lambda_{1} \rho \int_{0}^{1} q_{1}(s) d s+\lambda_{2} \rho \int_{0}^{1} q_{2}(s) d s\right)\right) \\
& \geq \tilde{\theta}\left(\omega R_{1}-\left(\lambda_{1} \rho \int_{0}^{1} q_{1}(s) d s+\lambda_{2} \rho \int_{0}^{1} q_{2}(s) d s\right)\right) \\
& \geq \lambda_{1} \rho \tilde{\theta} \int_{0}^{1} q_{1}(s) d s+\lambda_{2} \rho \tilde{\theta} \int_{0}^{1} q_{2}(s) d s \geq \tilde{N}, \quad t \in[\tilde{a}, \tilde{b}] .
\end{aligned}
$$

Thus, for any $(u, v) \in \partial K_{R_{1}}$, by (3.16)-(3.18), we have

$$
f_{1}\left(t,\left[u(t)-\varpi_{1}(t)\right]^{*},\left[v(t)-\varpi_{2}(t)\right]^{*}\right) \geq \wedge, \quad t \in[\tilde{a}, \tilde{b}] .
$$

Hence, for any $(u, v) \in \partial K_{R_{1}}$, by (3.19) and Lemma 2.3, we have

$$
\begin{aligned}
\left\|T_{1}(u, v)\right\|= & \max _{t \in[0,1]} \mid \lambda_{1} \int_{0}^{1} K_{1}(t, s)\left(f_{1}\left(s,\left[u(s)-\varpi_{1}(s)\right]^{*},\left[v(s)-\varpi_{2}(s)\right]^{*}\right)+q_{1}(s)\right) d s \\
& +\lambda_{2} \int_{0}^{1} H_{1}(t, s)\left(f_{2}\left(s,\left[u(s)-\varpi_{1}(s)\right]^{*},\left[v(s)-\varpi_{2}(s)\right]^{*}\right)+q_{2}(s)\right) d s \mid \\
\geq & \max _{t \in[0,1]} \lambda_{1} \int_{0}^{1} K_{1}(t, s)\left(f_{1}\left(s,\left[u(s)-\varpi_{1}(s)\right]^{*},\left[v(s)-\varpi_{2}(s)\right]^{*}\right)+q_{1}(s)\right) d s
\end{aligned}
$$




$$
\begin{aligned}
& \geq \min _{t \in[\tilde{a}, \tilde{b}]} \lambda_{1} \int_{\tilde{a}}^{\tilde{b}} \varrho t^{\alpha_{1}-1} s(1-s)^{\alpha_{1}-1} f_{1}\left(s,\left[u(s)-\varpi_{1}(s)\right]^{*},\left[v(s)-\varpi_{2}(s)\right]^{*}\right) d s \\
& \geq \lambda_{1} \tilde{\theta} \wedge \int_{\tilde{a}}^{\tilde{b}} \varrho s(1-s)^{\alpha_{0}-1} d s \geq R_{1}=\|(u, v)\| .
\end{aligned}
$$

Consequently,

$$
\|T(u, v)\|=\max \left\{\left\|T_{1}(u, v)\right\|,\left\|T_{2}(u, v)\right\|\right\} \geq R_{1}=\|(u, v)\| \quad \text { for any }(u, v) \in \partial K_{R_{1}} .
$$

Obviously, by the inequality

$$
\liminf _{y \rightarrow+\infty} \inf _{\substack{t \in[\tilde{a}, \tilde{b}] \subset(0,1) \\ x \in[0,+\infty)}} f_{1}(t, x, y)>\wedge
$$

in $\left(\mathbf{H}_{7}\right),(3.20)$ is still valid.

On the other hand, choose $\varepsilon_{i}>0$ such that

$$
\varepsilon_{i}=\left(3 \lambda_{i} \rho \int_{0}^{1} a_{i}(s) d s\right)^{-1}, \quad i=1,2 .
$$

Then, for the above $\varepsilon_{i}$, by the first inequality in $\left(\mathbf{H}_{7}\right)$, there exists $N^{\prime}>0$ such that for any $t \in[0,1]$, we have

$$
p_{i}(t, x, y) \leq \varepsilon_{i} x, \quad x \geq N^{\prime}, y \geq 0, i=1,2 .
$$

Then we have

$$
p_{i}(t, x, y) \leq \Phi+\varepsilon_{i} x, \quad t \in[0,1], x \geq 0, y \geq 0, i=1,2,
$$

where $\Phi=\max \left\{p_{i}(t, x, y): t \in[0,1], 0 \leq x \leq N^{\prime}, 0 \leq y \leq N^{\prime}, i=1,2\right\}$. Select

$$
R_{2} \geq \max \left\{2 R_{1},(\Phi+1)\left(\lambda_{1} \rho \int_{0}^{1}\left(a_{1}(s)+q_{1}(s)\right) d s+\lambda_{2} \rho \int_{0}^{1}\left(a_{2}(s)+q_{2}(s)\right) d s\right)\right\} .
$$

Assume

$$
K_{R_{2}}=\left\{(u, v) \in K:\|(u, v)\|<R_{2}\right\} .
$$

For any $(u, v) \in \partial K_{R_{2}}$, by (3.21) and Lemma 2.3, we have

$$
\begin{aligned}
\left\|T_{1}(u, v)\right\|= & \max _{t \in[0,1]} \mid \lambda_{1} \int_{0}^{1} K_{1}(t, s)\left(f_{1}\left(s,\left[u(s)-\varpi_{1}(s)\right]^{*},\left[v(s)-\varpi_{2}(s)\right]^{*}\right)+q_{1}(s)\right) d s \\
& +\lambda_{2} \int_{0}^{1} H_{1}(t, s)\left(f_{2}\left(s,\left[u(s)-\varpi_{1}(s)\right]^{*},\left[v(s)-\varpi_{2}(s)\right]^{*}\right)+q_{2}(s)\right) d s \mid \\
\leq & \max _{t \in[0,1]} \mid \lambda_{1} \int_{0}^{1} \rho t^{\alpha_{1}-1}\left(a_{1}(s) p_{1}\left(s,\left[u(s)-\varpi_{1}(s)\right]^{*},\left[v(s)-\varpi_{2}(s)\right]^{*}\right)+q_{1}(s)\right) d s \\
& +\lambda_{2} \int_{0}^{1} \rho t^{\alpha_{1}-1}\left(a_{2}(s) p_{2}\left(s,\left[u(s)-\varpi_{1}(s)\right]^{*},\left[v(s)-\varpi_{2}(s)\right]^{*}\right)+q_{2}(s)\right) d s \mid
\end{aligned}
$$




$$
\begin{aligned}
\leq & \lambda_{1} \rho \int_{0}^{1}\left(a_{1}(s)\left(\Phi+\varepsilon_{1}\left[u(s)-\varpi_{1}(s)\right]^{*}\right)+q_{1}(s)\right) d s \\
& +\lambda_{2} \rho \int_{0}^{1}\left(a_{2}(s)\left(\Phi+\varepsilon_{2}\left[u(s)-\varpi_{1}(s)\right]^{*}\right)+q_{2}(s)\right) d s \\
\leq & (\Phi+1)\left(\lambda_{1} \rho \int_{0}^{1}\left(a_{1}(s)+q_{1}(s)\right) d s+\lambda_{2} \rho \int_{0}^{1}\left(a_{2}(s)+q_{2}(s)\right) d s\right) \\
& +\lambda_{1} \rho \varepsilon_{1}\|u\| \int_{0}^{1} a_{1}(s) d s+\lambda_{2} \rho \varepsilon_{2}\|u\| \int_{0}^{1} a_{2}(s) d s \\
\leq & R_{2}=\|(u, v)\| .
\end{aligned}
$$

Similarly as (3.22), for any $(u, v) \in \partial K_{R_{2}}$, by (3.21) and Lemma 2.3, we also have

$$
\left\|T_{2}(u, v)\right\| \leq R_{2}=\|(u, v)\|
$$

Consequently, we have

$$
\|T(u, v)\|=\max \left\{\left\|T_{1}(u, v)\right\|,\left\|T_{2}(u, v)\right\|\right\} \leq R_{2}=\|(u, v)\| \quad \text { for any }(u, v) \in \partial K_{R_{2}} .
$$

It follows from the above discussion, (3.20), (3.23), Lemmas 2.6 and 2.7, that for any $\lambda_{1} \in$ $\left(\tilde{\lambda}_{1},+\infty\right), \lambda_{2} \in\left(\tilde{\lambda}_{2},+\infty\right), T$ has a fixed point $(u, v) \in \bar{K}_{R_{2}} \backslash K_{R_{1}}$ and $R_{1} \leq\|(u, v)\| \leq R_{2}$. Since $\|(u, v)\| \geq R_{1}$, by the same method as Theorem 3.3, we know that for any $\lambda_{1} \in\left(\tilde{\lambda}_{1},+\infty\right)$, $\lambda_{2} \in\left(\tilde{\lambda}_{2},+\infty\right)$, system (1.1) has at least one positive solution $(\tilde{u}, \tilde{v})$. Moreover, $(\tilde{u}, \tilde{v})$ satisfies $\tilde{u}(t) \geq \tilde{l} t^{\alpha_{1}-1}, \tilde{v}(t) \geq \tilde{l} t^{\alpha_{2}-1}, t \in[0,1]$. The proof is completed.

Remark 3.4 From the proof of Theorem 3.4, we know that the conclusion of Theorem 3.4 is valid if the second equality of condition $\left(\mathbf{H}_{7}\right)$ is replaced by

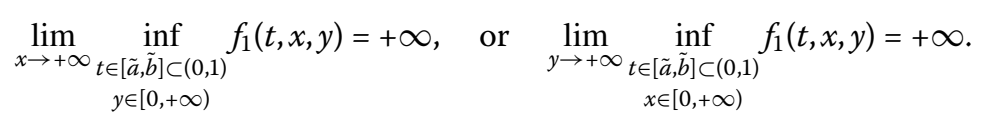

Remark 3.5 From the proof of Theorem 3.4, we know that the conclusion of Theorem 3.4 is valid if the second equality of condition $\left(\mathbf{H}_{7}\right)$ is replaced by

$$
\liminf _{x \rightarrow+\infty} \inf _{\substack{t \in[\tilde{a}, \tilde{b}] \subset(0,1) \\ y \in[0,+\infty)}} f_{2}(t, x, y)>\wedge, \quad \text { or } \quad \liminf _{y \rightarrow+\infty} \inf _{\substack{t \in[\tilde{a}, \tilde{b}] \subset(0,1) \\ x \in[0,+\infty)}} f_{2}(t, x, y)>\wedge
$$

where $\wedge$ is defined in Theorem 3.4.

Similarly as Remark 3.4, the conclusion of Theorem 3.4 is also valid if the second equality of condition $\left(\mathbf{H}_{7}\right)$ is replaced by

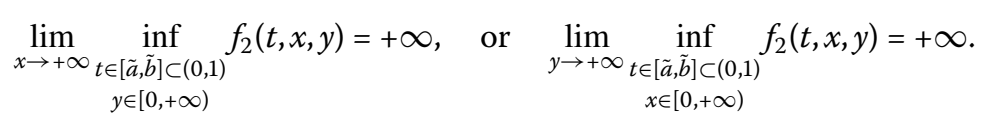




\section{Example}

Consider the fractional differential system

$$
\left\{\begin{array}{l}
D_{0^{+}}^{\frac{5}{2}} u(t)+\lambda_{1} f_{1}(t, u(t), v(t))=0, \\
D_{0^{+}}^{\frac{5}{2}} v(t)+\lambda_{2} f_{2}(t, u(t), v(t))=0, \quad 0<t<1, \\
u(0)=u^{\prime}(0)=0, \quad u(1)=\frac{1}{2} \int_{0}^{1} v(t) d t, \\
v(0)=v^{\prime}(0)=0, \quad v(1)=\int_{0}^{1} u(t) d t^{\frac{1}{2}},
\end{array}\right.
$$

where $\lambda_{i}>0(i=1,2)$ is a parameter, $\alpha_{1}=\alpha_{2}=\frac{5}{2}, \mu_{1}=\frac{1}{2}, \mu_{2}=1, A_{1}(t)=t, A_{2}(t)=t^{\frac{1}{2}}$. Then we have

$$
\begin{aligned}
& k_{1}=\int_{0}^{1} t^{\alpha_{2}-1} d A_{1}(t)=\int_{0}^{1} t^{\frac{3}{2}} d t=\frac{2}{5}>0 \\
& k_{2}=\int_{0}^{1} t^{\alpha_{1}-1} d A_{2}(t)=\int_{0}^{1} t^{\frac{3}{2}} d t^{\frac{1}{2}}=\frac{1}{2} \int_{0}^{1} t d t=\frac{1}{4}>0, \quad 1-\mu_{1} \mu_{2} k_{1} k_{2}=\frac{19}{20}>0 .
\end{aligned}
$$

So, condition $\left(\mathbf{H}_{0}\right)$ holds.

Next, in order to demonstrate the application of our main results obtained in Section 3, we choose two different sets of functions $f_{i}(t, x, y)(i=1,2)$ such that $f_{i}$ satisfies the conditions of Theorems 3.3 and 3.4.

Case 1. Let $f_{1}(t, x, y)=\frac{x^{2}+y^{2}}{\sqrt{t(1-t)}}+\ln t, f_{2}(t, x, y)=\frac{1+e^{x}+e^{y}}{\sqrt{t(1-t)}}+\ln (1-t),(t, x, y) \in(0,1) \times$ $[0,+\infty) \times[0,+\infty)$. Take $a_{1}(t)=a_{2}(t)=\frac{1}{\sqrt{t(1-t)}}, q_{1}(t)=-\ln t, q_{2}(t)=-\ln (1-t), p_{1}(t, x, y)=$ $x^{2}+y^{2}, p_{2}(t, x, y)=1+e^{x}+e^{y}$, then

$$
-q_{i}(t) \leq f(t, x, y) \leq a_{i}(t) p_{i}(t, x, y), \quad(t, x, y) \in(0,1) \times[0,+\infty) \times[0,+\infty), i=1,2 .
$$

By a direct calculation, we have

$$
\int_{0}^{1} a_{1}(t) d t=\int_{0}^{1} a_{2}(t) d t=\pi, \quad \int_{0}^{1} q_{1}(t) d t=\int_{0}^{1} q_{2}(t) d t=1 .
$$

So condition $\left(\mathbf{H}_{1}\right)$ holds.

In addition, choose $\left[\frac{1}{3}, \frac{2}{3}\right] \subset[0,1]$, we know

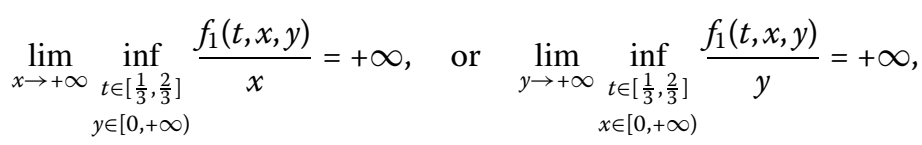

so condition $\left(\mathbf{H}_{6}\right)$ of Theorem 3.3 is satisfied.

Therefore, by Theorem 3.3, we obtain that system (4.1) has at least one positive solution provided $\lambda_{i}>0(i=1,2)$ is small enough.

Case 2. Let $f_{1}(t, x, y)=\frac{\sqrt{2}(x+y)^{\frac{1}{2}}}{\sqrt[4]{t^{3}(1-t)\left(1+t^{2}(1-t)\right)}}-\frac{2}{\sqrt{t}}, f_{2}(t, x, y)=\frac{1}{e^{x+y+1} \sqrt{t(1-t)}}-\frac{3}{\sqrt{1-t}},(t, x, y) \in(0,1) \times$ $[0,+\infty) \times[0,+\infty)$. Take $a_{1}(t)=\frac{\sqrt{2}}{\sqrt[4]{t^{3}(1-t)}}, a_{2}(t)=\frac{1}{\sqrt{t(1-t)}}, q_{1}(t)=\frac{2}{\sqrt{t}}, q_{2}(t)=\frac{3}{\sqrt{1-t}}, p_{1}(t, x, y)=$ $\frac{(x+y)^{\frac{1}{2}}}{1+t^{2}(1-t)}, p_{2}(t, x, y)=\frac{1}{e^{x+y+1}}$, then

$$
-q_{i}(t) \leq f(t, x, y) \leq a_{i}(t) p_{i}(t, x, y), \quad(t, x, y) \in(0,1) \times[0,+\infty) \times[0,+\infty), i=1,2 .
$$


By a direct calculation, we have

$$
\int_{0}^{1} a_{1}(t) d t=2 \pi, \quad \int_{0}^{1} a_{2}(t) d t=\pi, \quad \int_{0}^{1} q_{1}(t) d t=4, \quad \int_{0}^{1} q_{2}(t) d t=6 .
$$

\section{So condition $\left(\mathbf{H}_{1}\right)$ holds.}

In addition, choose $\left[\frac{1}{4}, \frac{3}{4}\right] \subset[0,1]$, we know

$$
\limsup _{x \rightarrow+\infty} \sup _{\substack{t \in[0,1] \\ y \in[0,+\infty)}} \frac{p_{i}(t, x, y)}{x}=0, \quad i=1,2
$$

and

$$
\begin{aligned}
& \liminf _{x \rightarrow+\infty} \inf _{t \in\left[\frac{1}{4}, \frac{3}{4}\right]} f_{1}(t, x, y)=+\infty, \quad \text { or } \quad \liminf _{y \rightarrow+\infty} \inf _{t \in\left[\frac{1}{4}, \frac{3}{4}\right]} f_{1}(t, x, y)=+\infty \text {, } \\
& y \in[0,+\infty) \quad x \in[0,+\infty)
\end{aligned}
$$

so condition $\left(\mathbf{H}_{7}\right)$ of Theorem 3.4 is satisfied.

Therefore, by Theorem 3.4, we obtain that system (4.1) has at least one positive solution provided $\lambda_{i}>0(i=1,2)$ is sufficiently large.

\section{Competing interests}

The authors declare that they have no competing interests.

\section{Authors' contributions}

The study was carried out in collaboration between all authors. YW completed the main part of this paper and gave two examples; LSL and YHW corrected the main theorems and polished the manuscript. All authors read and approved the final manuscript.

\section{Author details}

'School of Mathematical Sciences, Qufu Normal University, Qufu, Shandong 273165, People's Republic of China. ${ }^{2}$ School of Science, Linyi University, Linyi, Shandong 276000, People's Republic of China. ${ }^{3}$ Department of Mathematics and Statistics, Curtin University of Technology, Perth, WA6845, Australia.

\section{Acknowledgements}

The first and second authors were supported financially by the National Natural Science Foundation of China (11371221) the Specialized Research Foundation for the Doctoral Program of Higher Education of China (20123705110001) and the Program for Scientific Research Innovation Team in Colleges and Universities of Shandong Province. The third author was supported financially by the Australia Research Council through an ARC Discovery Project Grant.

\section{Received: 2 July 2014 Accepted: 2 October 2014 Published: 14 Oct 2014}

\section{References}

1. Amann, H: Parabolic evolution equations and nonlinear boundary conditions. J. Differ. Equ. 72, $201-269$ (1988)

2. Mehmeti, FA, Nicaise, S: Nonlinear interaction problems. Nonlinear Anal. 20, 27-61 (1993)

3. Pao, CV: Finite difference reaction-diffusion systems with coupled boundary conditions and time delays. J. Math. Anal. Appl. 272, 407-434 (2002)

4. Wang, S: Doubly nonlinear degenerate parabolic systems with coupled nonlinear boundary conditions. J. Differ. Equ. $182,431-469(2002)$

5. Leung, A: A semilinear reaction-diffusion prey-predator system with nonlinear coupled boundary conditions: equilibrium and stability. Indiana Univ. Math. J. 31, 223-241 (1982)

6. Aronson, DG: A comparison method for stability analysis of nonlinear parabolic problems. SIAM Rev. 20, 245-264 (1978)

7. Salem, HAH: On the existence of continuous solutions for a singular system of non-linear fractional differential equations. Appl. Math. Comput. 198, 445-452 (2008)

8. Goodrich, CS: Existence of a positive solution to systems of differential equations of fractional order. Comput. Math. Appl. 62, 1251-1268 (2011)

9. Wang, GT, Agarwal, RP, Cabada, A: Existence results and the monotone iterative technique for systems of nonlinear fractional differential equations. Appl. Math. Comput. 25, 1019-1024 (2012)

10. Li, R, Zhang, HQ, Tao, H: Unique solution of a coupled fractional differential system involving integral boundary conditions from economic model. Abstr. Appl. Anal. 2013, Article ID 615707 (2013) 
11. Henderson, J, Luca, R: Positive solutions for a system of nonlocal fractional boundary value problems. Fract. Calc. Appl. Anal. 16(4), 985-1008 (2013)

12. Henderson, J, Luca, R: Existence and multiplicity of positive solutions for a system of fractional boundary value problems. Bound. Value Probl. 2014, Article ID 60 (2014)

13. Zhang, HN, Gao, WJ: Existence and uniqueness results for a coupled system of nonlinear fractional differential equations with antiperiodic boundary conditions. Abstr. Appl. Anal. 2014, Article ID 463517 (2014)

14. Wang, JH, Xiang, HJ, Liu, ZG: Positive solution to nonzero boundary values problem for a coupled system of nonlinear fractional differential equations. Int. J. Differ. Equ. 2010, Article ID 186928 (2010)

15. Yang, WG: Positive solutions for a coupled system of nonlinear fractional differential equations with integral boundary conditions. Comput. Math. Appl. 63, 288-297 (2012)

16. Miller, KS, Ross, B: An Introduction to the Fractional Calculus and Fractional Differential Equations. Wiley, New York (1993)

17. Podlubny, I: Fractional Differential Equations. Mathematics in Science and Engineering, vol. 198. Academic Press, New York (1999)

18. Yuan, CJ: Multiple positive solutions for $(n-1,1)$-type semipositone conjugate boundary value problems of nonlinear fractional differential equations. Electron. J. Qual. Theory Differ. Equ. 2010, 36 (2010)

19. Guo, DJ, Lakshmikantham, V: Nonlinear Problems in Abstract Cones. Academic Press, New York (1988)

10.1186/1687-1847-2014-268

Cite this article as: Wang et al.: Positive solutions for a class of higher-order singular semipositone fractional differential systems with coupled integral boundary conditions and parameters. Advances in Difference Equations 2014, 2014:268

\section{Submit your manuscript to a SpringerOpen ${ }^{\odot}$ journal and benefit from:}

- Convenient online submission

- Rigorous peer review

- Immediate publication on acceptance

Open access: articles freely available online

- High visibility within the field

- Retaining the copyright to your article 